\title{
Ueber allgemeine Thetaformeln.
}

\author{
Von
}

A. Krazer in Strassburg i. E.

Herr Prym und ich*) haben gezeigt, wie man zu Thetaformeln von allgemeinem Charakter dadurch gelangen kann, dass man in der ein vorgelegtes Product von $n$ Thetafunctionen von je $p$ Veränderlichen darstellenden $n p$-fach unendlichen Reihe an Stelle der bisherigen Summationsbuchstaben neue vermittelst einer linearen Substitution mit rationalen Coefficienten einführt. Diese unsere Untersuchungen bedürfen nach mehreren Richtungen der Ergänzung.

1. Die von Herrn Prym und mir angewandte Methode der Umformung unendlicher Reihen ist, wie wir übrigens schon bei Beginn unserer gemeinschaftlichen Untersuchungen bemerkt haben, nicht anf Thetareihen beschränkt, sondern auf ganz beliebige unendliche Reihen anwendbar. - Im ersten Abschnitte der vorliegenden Abhandlung wird dieses Princip der Umformung unendlicher, insbesondere mehrfach unendlicher Reihen durch Einführung neuer Summationsbuchstaben vermittelst einer linearen Substitution mit rationalen Coefficienten, von allem Zufälligen, was ihm bei der Anwendung anf Thetareihen anhaftet, entkleidet, in der vollen Allgemeinheit dargestellt $\left.{ }^{*}\right)$.

2. In den Formeln, welche die soeben genannten Umformungen unendlicher Reihen zum Ausdrucke bringen, kommen gewisse positive ganze Zahlen vor, welche als die Anzahlen der Lösungen von Systemen linearer Congruenzen auftreten. Auf die Bestimmung dieser Zahlen,

*) Prym, Ableitung einer allgemeinen Thetaformel. Acta math. Bd. 3 (1883) pag. 216.

Krazer und Prym, Neue Grundlagen einer Theorie der allgemeinen Thetafunctionen. Leipzig 1892.

**) Mit dieser Umformung unendlicher Reihen beschäftigt sich auch eine inzwischen erschienene Abhandlung des Herrn Huebner, Ueber die Umformung unendlicher Reihen und Producte mit Beziehung auf die Theorie der elliptischen Fanctionen (Progr. Königsberg 1891), deren Resultate aber nur im Falle einfach unendlicher Reihen mit den hier angegebenen übereinstimmen. 
die zuerst von Henry St. Smith*) und später aber unabhängig davon von Herrn Frobenius**) angegeben wurde, ist von Herrn Prym und mir nirgendwo eingegangen worden. Sie wird im zweiten Abschnitte der vorliegenden Abhandlung in Arlehnung an die soeben citirte Abbandlung des Herrn Frobenius, aber auf einem von dem dortigen verschiedenen Wege durchgeführt.

3. In den Untersuchungen von Herrn Prym und mir ist die Frage nach der allgemeinsten linearen Substitution mit rationalen Coefficienten, durch welche ein gegebenes Product von $n$ Thetafunctionen von je $p$ Veränderlichen in ein Aggregat solcher Producte äbergeführt wird, und damit die Frage nach der allgemeinsten einschlägigen Thetaformel nirgendwo beantwortet worden. Nur die von uns angegebene Umformung einer einzelnen Thetareibe**) ist die allgemeinste derartige Umformung; bei der Umformung eines Products von mehreren Thetafunctionen $\uparrow$ ) dagegen legten wir der Substitution, vermittelst welcher an Stelle der bisherigen $n p$ Summationsbuchstaben $m_{\mu}^{(\varrho)}\left(\begin{array}{l}\varrho=1,2, \ldots, n \\ \mu=1,2, \ldots, p\end{array}\right)$ $n p$ neue Summationsbuchstaben $n_{\mu}^{(\varrho)}\left(\begin{array}{l}\varrho=1,2, \ldots, n \\ \mu=1,2, \ldots, p\end{array}\right)$ eingefübrt werden, von vornherein freiwillig die Beschränkung auf, dass sie in jeder ihrer Gleichungen nur Grössen $m$ and $n$ mit demselben unteren Index $\mu$ enthalte. Es wurden also bis jetzt neben den Substitutionen zur Umformung einer einzelnen Thetafnnction (Substitutionen $E$ ) nur eine ganz besondere Art von Substitutionen zur Umformung eines Productes von mehreren Thetafunctionen (Substitutionen $D$ ) in Betracht gezogen, und wenn wir auch als wahrscheinlich ansahen, dass aus diesen beiden Arten von Substitutionen die allgemeinste Substitution $S$, welche ein Product von $n$ Thetafunctionen von je $p$ Veränderlichen in ein Aggregat von solchen Producten überführt, zusammengesetzt werden könne, so war es uns doch nicht gelungen, einen Beweis für die Richtigkeit dieser Vermuthung zu erbringen. Dieser Beweis wird im dritten Abschnitte der vorliegenden Abhandlung geführt.

4. Von Herrn Prym and mir ist die Stellung unserer Thetaformeln zu den von andern Autoren veröffentlichten nirgendwo erörtert worden. Dieser Aufgabe ist der vierte Abschnitt der gegenwärtigen Abhandlnng gewidmet. Es zeigt sich dabei, dass von den den Substitutionen $E$ entsprechenden Thetaformeln früher nur ganz specielle

*) Smith, On Systems of Linear Indeterminate Equations and Congruences (1861). Coll. math. Papers, Vol. 1, pag. 367.

*) Frobenius, Theorie der linearen Formen mit ganzen Coefficienten. J. für Matb. Bd. 86 (1879), pag. 146.

***) Krazer und Prym, Neue Grundlagen etc. pag. 70.

†) Krazer und Prym, a. a. O. pag. 16. 
Fälle bekannt gewesen waren. Etwas anders liegt die Sache für die zu den Substitutionen $D$ gehörigen Thetaformeln. Von ihnen war die dem Werthe $r=1$ entsprechende Formel für Producte von je zwei Thetafunctionen bereits 1854 von Schröter seinen Untersuchungen über die Modulargleichungen zu Grunde gelegt und auf Producte von beliebig vielen Thetafunctionen 1866 von Herrn Gordan ausgedehnt worden. Ohne damals diese Formel des Herrn Gordan za kennen, gelangten wir 1884 in naturgemässer Fortsetzung früherer Untersuchungen*) zur Verallgemeinerung der Schröter'schen Formel. Bevor wir die ron uns gefundene Formel veröffentlichten **) war auf anderem Wege und gleichfalls unabhängig von Herrn Gordan Herr Krause zu der nämlichen Formel gekommen und hatte dieselbe unter dem Namen ,Additionstheorem zwischen Thetafunctionen mit verschiedenen Moduln" Untersuchungen über die Transformation der Thetafunctionen zu Grunde gelegt.

\section{Erster Abschnitt.}

\section{Ein allgemeines Princip der Umformung unendlicher, insbesondere mehrfach unendlicher Reihen.}

Es sei gegeben eine $q$-fach unendliche absolut convergente Reihe:

$$
W=\sum_{m_{1}, \cdots, m_{q}}^{-\infty, \ldots,+\infty} f\left(m_{1}|\cdots| m_{q}\right)
$$

deren allgemeines Glied $f\left(m_{1}|\cdots| m_{q}\right)$ im Uebrigen eine beliebige Function der Summationsbuchstaben $m_{1}, \ldots, m_{q}$ und anderer Grössen, Variablen und Constanten, sein möge, und bei der die Summation so auszuführen ist, dass jede der $q$ Grössen $m$ unabhängig von den anderen die Reihe der ganzen Zahlen von $-\infty$ bis $+\infty$ durchläuft, sodass an Stelle des Systems der $q$ Summationsbuchstaben $m_{1}, \ldots, m_{q}$ jede Variation mit Wiederholung zur $q^{\text {ten }}$ Classe tritt, die man aus den überhaupt existirenden ganzen Zahlen als Elementen bilden kann. In dieser $q$-fach unendlichen Reihe führe man jetzt an Stelle der bisherigen Summationsbuchstaben $m_{1}, \ldots, m_{q}$ neue $n_{1}, \ldots, n_{q}$ ein mit Hülfe einer linearen Substitution von der Gestalt:

$$
r m_{\mu}=\sum_{\nu=1}^{q} a_{\mu \nu} n_{\nu}, \quad(\mu=1,2, \ldots, q)
$$

*) Prym, Ableitung einer allgemeinen Thetaformel. Acta math. Bd. 3 (1883), pag. 216.

**) Eine Mittheilung der Formel, aber ohne die Absicht der Veröffentlichung geschah an die Münchener Akademie am 11. August 1885. 
bei der $r$ eine positive ganze Zahl, die a ganze Zahlen mit nicht verschwindender Determinante sind. Man erhält dann, wenn man den Ausdruck, in den das allgemeine Glied $f\left(m_{1}|\cdots| m_{q}\right)$ durch Einführung der Grössen $n$ übergeht, mit $g\left(n_{1}|\cdots| n_{q}\right)$ bezeichnet, also:

$$
f\left(m_{1}|\cdots| m_{q}\right)=g\left(n_{1}|\cdots| n_{q}\right)
$$

setzt:

$$
W=\sum_{n_{1}, \cdots, n_{q}} g\left(n_{1}|\cdots| n_{q}\right)
$$

und es ist die Frage auf die Alles ankommt, die, wie hier über die $n$ summirt werden muss. Diese Frage ist vorerst folgendermassen zu beantworten. Bezeichnet man die Determinante der $q^{2}$ Zahlen $a_{\mu \nu}$ mit $\Delta$ und die Adjuncte von $a_{\mu \nu}$ in dieser Determinante mit $\alpha_{\mu \nu}$, so folgt aus den Gleichungen (2) durch Auflösung nach den $n$ als Unbekannten:

$$
n_{\nu}=\frac{r}{\Delta} \sum_{\mu=1}^{q} \alpha_{\mu \nu} m_{\mu}
$$$$
(\nu=1,2, \ldots, q)
$$

und es muss daher die auf der rechten Seite von (4) angedeutete Summation nach den $n$ in der Weise ausgeführt werden, dass man an Stelle des Systems der $q$ Summationsbuchstaben $n_{1}, \ldots, n_{q}$ ein jedes der Werthesysteme und jedes einmal setzt, welche sich aus den Gleichungen (5) ergeben, wenn man darin an Stelle des Systems der $q$ Buchstaben $m_{1}, \ldots, m_{q}^{3}$ eine jede der Variationen mit Wiederholung zur $q^{\text {ten }}$ Classe aus den überhaupt existirenden ganzen Zahlen als Elementen treten lässt.

Zur directen Bestimmung dieser Werthesysteme, von denen man jedenfalls sagen kann, dass keine zwei unter ihnen mit einander übereinstimmen, muss das System der durch die Gleichungen (ó) als Functionen der ganzen Zahlen $m$ definirten Grössen $n$ genau untersucht werden. Zu dem Ende bezeichne man mit $\varrho_{\mu}$ den kleinsten positiven Rest der Zahl $m_{\mu}$ nach dem Modul $\Delta$ und setze:

$$
m_{\mu}=\Delta m_{\mu}^{\prime}+\varrho_{\mu} \text {. }
$$

$$
(\mu=1,2, \ldots, q)
$$

Führt man diese Ausdrücke in die Gleichungen (5) ein, so zerfällt jede Grösse $n_{\nu}$ in einen ganzzahligen Theil $n_{\nu}^{\prime}$ und einen Bruch, in der Form:

$$
n_{\nu}=n_{\nu}^{\prime}+\frac{r}{\Delta} \sum_{\mu=1}^{q} \alpha_{\mu \nu} \varrho_{\mu} . \quad(\nu=1,2, \ldots, q)
$$

Die $n^{\prime}$ sind ganze 'Zahlen von besonderer Art; anstatt auf ihre Ausdrücke in den $m^{\prime}$ einzugehen, bemerke man, dass für die $n^{\prime}$ jedenfalls nur solche ganze Zahlen auftreten, welche nach Einführung der ihnen 
entsprechenden Ausdrücke (7) in die Gleichungen (2) für die $m$ ganze Zahlen liefern. Setzt man aber aus (7) in (2) ein, so folgt:

$$
r m_{\mu}=\sum_{\nu=1}^{q} a_{\mu \nu} n_{\nu}^{\prime}+r \varphi_{\mu}, \quad(\mu=1,2, \ldots, q)
$$

und man erhält daher für die ganzen Zahlen n' die Bedingung, dass:

$$
\sum_{\nu=1}^{q} a_{\mu \nu} n_{\nu}^{\prime} \equiv 0(\bmod . r) \quad(\mu=1,2, \ldots, q)
$$

sei. Sind diese Bedingungen erfüllt, dann entsprechen den zu solchen $n^{\prime}$ gemäss den Gleichungen (7) gehörigen Grössen $n$ in der That ganze Zahlen $m$. Bildet man daher die Summe:

$$
\sum_{\varrho_{1}, \cdots, \varrho_{q}}^{0,1, \cdots, \nabla-1} \sum_{n_{1}^{\prime}, \cdots, n_{q}^{\prime}}^{-\infty, \cdots,+\infty} g\left(n_{1}^{\prime}+\frac{\bar{\rho}_{1}}{\Delta}|\cdots| n_{q}^{\prime}+\frac{\overline{\bar{\rho}}_{q}}{\Delta}\right),
$$

bei der zur Abkürzung:

$$
\overline{\bar{\varrho}}_{\nu}=r \sum_{\mu=1}^{q} \alpha_{\mu \nu} \varrho_{\mu} \quad(\nu=1,2, \ldots, q)
$$

gesetzt ist, und bei der, indem $\nabla$ den absoluten Werth von $\Delta$ bezeichnet, über jedes $\rho$ frei von 0 bis $\nabla-1$ summirt wird, für das System der $q$ Summationsbuchstaben $n^{\prime}$ aber nur jene aus ganzen Zahlen gebildeten Variationen mit Wiederholung zur $q^{\text {ten }}$ Classe zu treten haben, welche in ihren Elementen den Congruenzen (9) genügen, so enthält diese Summe nach dem soeben Bemerkten alle Glieder der Summe (4) und keine anderen Glieder; und es frägt sich nur noch, ob sie auch jedes Glied der Summe (4) nur einmal oder ob sie es mehrere Male enthält, oder, was auf dasselbe hinauskommt, ob alle Glieder der Summe (10) von einander verschieden, oder ob sie theilweise einander gleich sind. Um diese Frage zu entscheiden, greife man ein bestimmtes Glied der Summe (10) heraus; es ist bestimmt durch gewisse den Congruenzen (9) genügende ganze Zahlen $n^{\prime}$ und gewisse positive ganze Zahlen $\rho$ aus der Reihe $0,1, \ldots, \nabla-1$. Soll ein anderes Glied, charakterisirt durch andere Zahlen $n^{\prime \prime}$ and $\sigma$ ihm gleich sein, so ist dazu nothwendig und hinreichend, dass für $\nu=1,2, \ldots, q$ :

$$
n_{\nu}^{\prime}+\frac{r}{\Delta} \sum_{\mu=1}^{g} \alpha_{\mu \nu} \varrho_{\mu}=n_{\nu}^{\prime \prime}+\frac{r}{\Delta} \sum_{\mu=1}^{q} \alpha_{\mu \nu} \sigma_{\mu}
$$

sei; dann muss aber jedenfalls: 


$$
r \sum_{\mu=1}^{q} \alpha_{\mu \nu}\left(\rho_{\mu}-\sigma_{\mu}\right) \equiv 0(\bmod . \nabla) \quad(\nu=1,2, \ldots, q)
$$

sein; ist umgekehrt diese Bedingung erfüllt und setzt man:

$$
r \sum_{\mu=1}^{q} \alpha_{\mu \nu} \varrho_{\mu}=r \sum_{\mu=1}^{q} \alpha_{\mu \nu} \sigma_{\mu}+\Delta g_{\nu} \quad(\nu=1,2, \ldots, q)
$$

so braucht man nur:

$$
n_{v}^{\prime \prime}=n_{v}^{\prime}+g_{v}
$$$$
(\nu=1,2, \ldots, q)
$$

zu nehmen, dann sind in der That die beiden Glieder der Summe einander gleich. So oft also die Congruenzen:

$$
r \sum_{\mu=1}^{q} \alpha_{\mu \nu} x_{\mu} \equiv 0(\bmod . \nabla) \quad(\nu=1,2, \ldots, q)
$$

durch Zahlen $x$ aus der Reihe $0,1, \ldots, \nabla-1$ befriedigt werden können, so oft kehrt jedes Glied der Summe (10) bei weiterem Fortgange der Summation wieder. Heisst man daher diese Anzahl $\sigma^{\prime}$, so ist die Summe (10) das $\sigma^{\prime}$-fache der Summe $W$ und man hat:

$$
W=\frac{1}{\sigma^{\prime}} \sum_{\varrho_{1}, \cdots, \varrho_{q}}^{0,1, \cdots, \nabla-1} \sum_{n_{1}^{\prime}, \cdots, n_{q}^{\prime}}^{-\infty, \cdots,+\infty} g\left(n_{1}^{\prime}+\frac{\overline{\bar{\rho}}_{1}}{\Delta}|\cdots| n_{q}^{\prime}+\frac{\overline{\overline{\rho_{q}}}}{\Delta}\right) .
$$

In dieser Gleichung bedeutet also $\sigma^{\prime}$ die Anzahl der Lösungen - um anzugeben, dass es sich dabei nur um jene Lösungen handelt, die aus Zahlen der Reihe $0,1, \cdots, \nabla-1$ gebildet sind, sei genauer gesagt "Normallösungen" - des Congruenzensystems (12), über jedes $\varrho$ ist frei zu summiren von 0 bis $\nabla-1$, über die $n^{\prime}$ von $-\infty$ bis $+\infty$, jedoch dürfen hier nur jene Zahlensysteme genommen werden, welche die Congruenzen (9) erfüllen. Von dieser Beschränkung der Summation kann man sich aber leicht auf folgende Weise befreien.

Multiplicirt man das allgemeine Glied der Summe in (13) mit einem Factor, der den Werth 1 hat, wenn die $n^{\prime}$ solche ganze Zahlen sind, die den Congrnenzen (9) genügen, dagegen den Werth Null, wenn die Zahlen $n^{\prime}$ diesen Congruenzen nicht genügen, so wird durch Einschiebung dieses Factors $F$, der in seiner Wirksamkeit mit dem Dirichlet'schen discontinuirlichen Factor der Integralrechnung zu vergleichen ist, zunächst der Werth der Summe (13) nicht geändert; nachdem er aber eingeschoben ist, darf jetzt die Beschränkung der Summation nach den $n^{\prime}$ einfach weggelassen werden, und man darf schreiben: 


$$
W=\frac{1}{\sigma^{\prime}} \sum_{\rho_{1}, \cdots, \rho_{q}}^{0,1, \cdots, \nabla-1} \sum_{n_{1}, \cdots, n_{q}}^{-\infty, \cdots,+\infty} F \cdot g\left(n_{1}+\frac{\overline{\overline{e_{1}}}}{\Delta}|\cdots| n_{q}+\frac{\overline{\bar{e}_{q}}}{\Delta !}\right),
$$

wo jetzt über jedes $\rho$ frei von 0 bis $\nabla-1$ und über jedes $n$ frei von $-\infty$ bis $+\infty$ summirt wird. Es handelt sich jetzt nur noch um die Bildung eines solchen Factors $F$. Beachtet man aber, dass die Grösse:

$$
f_{\mu}=\sum_{\sigma_{\mu}=0}^{r-1} e^{\frac{2 \pi i}{r} \sum_{\nu=1}^{q}\left(a_{\mu \nu} n_{\nu}\right) \sigma_{\mu}}
$$

den Werth $r$ besitzt, wenn die Zahlen $n$ die Congruenz:

$$
\sum_{\nu=1}^{q} a_{\mu \nu} n_{\nu} \equiv 0(\bmod . r)
$$

erfüllen, dagegen den Werth 0 , wenn sie dies nicht thun, so sieht man sofort, dass:

$$
F=\frac{f_{1} f_{2} \ldots f_{q}}{r^{q}}=\frac{1}{r^{q}} \sum_{\sigma_{1}, \cdots, \sigma_{q}}^{0,1, \ldots, r-1} e^{\frac{2 \pi i}{r} \sum_{\mu=1}^{q} \sum_{v=1}^{q} a_{\mu \nu} n_{\nu} \sigma_{\mu}}
$$

ein Factor der vorher verlangten Art ist. Setzt man aber diesen Ausdruck an Stelle von $F$ in (14) ein und vertauscht noch unter der Voraussetzung der absoluten Convergenz der neuen unendlichen Reihen die Summationsordnung, so erhält man die Gleichung:

$$
\begin{gathered}
W=\frac{1}{r^{q} \sigma^{\prime}} \sum_{\underline{a}_{1}, \cdots, o_{q}}^{0,1, \cdots, \nabla-1} \sum_{\sigma_{1}, \cdots, \sigma_{q}}^{0,1, \cdots, r-1} \\
{\left[\sum_{n_{1}, \cdots, n_{q}}^{-\infty, \cdots,+\infty} e^{\frac{2 \pi i}{r} \sum_{\mu=1}^{q} \sum_{q=1}^{q} a_{\mu}, \sigma_{\mu n v}} g\left(n_{1}+\frac{\overline{\underline{e}}_{1}}{\Delta}|\cdots| n_{q}+\frac{\overline{\rho_{q}}}{\Delta}\right)\right],}
\end{gathered}
$$

welche die gewünschte Umformung der gegebenen unendlichen Reihe darstellt, und der man schliesslich, weil:

$$
\sum_{\nu=1}^{q} a_{\mu \nu} \overline{\bar{\varrho}}_{\nu}=r \Delta \varrho_{\mu} \quad(\mu=1,2, \ldots, q)
$$


und in Folge dessen für alle ganzzahligen $\rho$ und $\sigma$

$$
e^{\frac{2 \pi i}{r}} \sum_{\mu=1}^{q} \sum_{\nu=1}^{q} a_{\mu \nu} \sigma_{\mu} \frac{\overline{\bar{o}}_{\nu}}{\Delta}=1
$$

ist, die für die Anwendungen bequemere Form:

$$
\begin{gathered}
W=\frac{1}{r^{q} \sigma^{\prime}} \sum_{\rho_{1}, \cdots, \rho_{q}}^{0,1, \cdots, \nabla-1} \sum_{\sigma_{1}, \cdots, \sigma_{q}}^{0,1, \cdots, r-1} \\
{\left[\sum_{n_{1}, \cdots, n_{q}}^{-\infty, \cdots+\infty} e^{\frac{2 \pi i}{r} \sum_{\nu=1}^{q} \bar{\sigma}_{\nu}\left(n_{\nu}+\frac{\overline{\bar{\varphi}_{\nu}}}{\Delta}\right)} g\left(n_{1}+\frac{\overline{\bar{\rho}_{q}}}{\Delta}|\cdots| n_{q}+\frac{\overline{\overline{\rho_{q}}}}{\Delta}\right)\right]}
\end{gathered}
$$

geben kann, bei der noch zur Abkürzung:

gesetzt ist.

$$
\sum_{\mu=1}^{q} a_{\mu \nu} \sigma_{\mu}=\bar{\sigma}_{\nu} \quad(\nu=1,2, \ldots, q)
$$

Bei Betrachtung der gewonnenen Endformel wird man zunächst das Resultat bemerken, dass die gegebene unendliche Reihe in eine Summe mehrerer unendlicher Reihen übergeführt wurde; bei genauerer Betrachtung des Ganges der Untersuchung erkennt man sodann, dass dieses Resultat einmal durch gruppenweises Zusammenfassen der Glieder der gegebenen Reihe zu Theilreihen, dann aber weiter durch Einschieben von Gruppen neuer Glieder, die zusammen den Werth Null haben, erreicht wurde. Aus dem letzteren Umstande erkennt man auch, dass auf die am Schlusse eingeführte Bedingung der absoluten Convergenz der neuen unendlichen Reihen nicht verzichtet werden kann, da sie nicht eine Folge der absoluten Convergenz der ursprünglichen Reihe ist.

Obwohl die Umformung (18) ihre wahre Bedeutung erst für mehrfach unendliche Reihen erlangt, so ist sie doch auch auf einfach unendliche Reihen anwendbar, und es liefern hier die beiden einfachsten Substitutionen $m=q n$ und $r m=n$ zwei Umformungen einer einfach unendlichen Reihe, bei denen die beiden oben genannten Processe des gruppenweisen Zusammenfassens der Glieder der gegebenen Reihe zu Theilreihen und des Einschiebens von Gruppen neuer Glieder mit der Summe Null einzeln auftreten und daher besonders klar erkennbar sind. Es entspricht nämlich der Substitution 


$$
\begin{aligned}
& \sum_{m=-\infty}^{+\infty} f(m)=\sum_{\varrho=0}^{q-1}\left(\sum_{n=-\infty}^{+\infty} g\left(n+\frac{\rho}{q}\right)\right)=\sum_{\varrho=0}^{q-1}\left(\sum_{n=-\infty}^{+\infty} f(q n+\varrho)\right) \\
& =[f(0)+f(q)+f(2 q)+\cdots] \\
& +[f(1)+f(q+1)+f(2 \dot{q}+1)+\cdots] \\
& +[f(2)+f(q+2)+f(2 q+2)+\cdots] \\
& +[f(q-1)+f(2 q-1)+f(3 q-1)+\cdots] *),
\end{aligned}
$$

während der Substitution

die Umformung;

$$
r m=n
$$

$$
\begin{gathered}
\sum_{m=-\infty}^{+\infty} f(m)=\frac{1}{r} \sum_{\sigma=0}^{r-1}\left(\sum_{n=-\infty}^{+\infty} e^{\frac{2 \pi i}{r} \sigma n} g(n)\right)=\frac{1}{r} \sum_{\sigma=0}^{r-1}\left(\sum_{n=-\infty}^{+\infty} e^{\frac{2 \pi i}{r} \sigma n} f\left(\frac{n}{r}\right)\right) \\
=\frac{1}{r}\left\{\left[\begin{array}{lll}
f(0)+ & f\left(\frac{1}{r}\right)+ & f\left(\frac{2}{r}\right)+\cdots
\end{array}\right]\right. \\
+\left[\begin{array}{lll}
f(0)+\tau & f\left(\frac{1}{r}\right)+\tau^{2} & f\left(\frac{2}{r}\right)+\cdots
\end{array}\right] \\
+\left[\begin{array}{lll}
f(0)+\tau^{2} & f\left(\frac{1}{r}\right)+\tau^{4} & f\left(\frac{2}{r}\right)+\cdots
\end{array}\right] \\
\cdot \cdot \cdot \cdot \cdot \cdot \cdot \cdot \cdot \cdot \cdot \cdot \cdot]
\end{gathered}
$$

entspricht, bei der zur Abkürzung:

gesetzt ist.

$$
\tau=e^{\frac{2 \pi i}{r}}
$$

\section{Zweiter Abschnitt.}

Ueber die Anzahl $s$ der Normallösungen eines Systems linearer Congruenzen.

Es seien mit $a_{\mu \nu}\left(\begin{array}{l}\mu=1,2, \ldots, p \\ \nu=1,2, \ldots, q\end{array}\right) p q$ beliebige ganze Zahlen, mit $r$ eine positive ganze Zahl bezeichnet; jedes System von $q$ ganzen Zahlen $x_{1}, x_{2}, \ldots, x_{q}$, welches gleichzeitig den $p$ Congruenzen:

$$
\sum_{\nu=1}^{q} a_{\mu \nu} x_{\nu} \equiv 0(\bmod . r) \quad(\mu=1,2, \ldots, p)
$$

*) Dabei sind, ebenso wie beim zweiten Beispiele, die den negativen Werthen des Summationsbuchstabens $n$ entsprechenden Glieder der Uebersichtlichkeit wegen unberücksichtigt gelassen. 
genügt, heisst eine Lösung dieses Congruenzensystems; Normallösungen aber sollen unter diesen unbegrenzt vielen Lösungen diejenigen genannt werden, welche ausschliesslich von Zahlen aus der Reibe $0,1, \ldots, r-1$ gebildet sind. Die Anzahl $s$ dieser Normallösungen ist dann jedenfalls eine endliche und zwat ist $s \overline{\overline{<}} r^{2}$. Es handelt sich im Folgenden wesentlich um die Bestimmung dieser Zahl $s$.

\section{1.}

Zunächst kann man ohne Mühe für die Zahl s einen analytischen Ausdruck anschreiben. Genügen nämlich die Zahlen $x_{1}, x_{2}, \ldots, x_{q}$ der Congruenz:

$$
\sum_{v=1}^{q} a_{\mu \nu} x_{\nu} \equiv 0(\bmod . r),
$$

wo $\mu$ irgend eine Zahl aus der Reihe $1,2, \ldots, p$ bezeichne, so besitzt der Ausdruck:

$$
f_{\mu}\left(x_{1}, \cdots \mid x_{q}\right)=f_{\mu}=\sum_{y_{\mu}=0}^{r-1} e^{\frac{2 \pi i}{r}\left(\sum_{\nu=1}^{q} a_{\mu v} x_{\nu}\right) y_{\mu}}
$$

den Werth $r$; genügen dagegen die Zahlen $x$ der angeschriebenen Congruenz nicht, so besitzt $f_{\mu}$ den Werth Null. Daraus folgt sofort, dass der Ausdruck:

$$
F\left(x_{1}|\cdots| x_{4}\right)=\frac{f_{1} f_{2} \ldots f_{p}}{r^{p}}=\frac{1}{r^{p}} \sum_{y_{1}, \cdots, y_{p}}^{0,1, \cdots, r-1} e^{\frac{2 \pi_{i}}{r} \sum_{\mu=1}^{p} \sum_{\nu=1}^{q} a_{\mu \nu} x_{\nu} y_{\mu}}
$$

für jedes Zahlensystem $x_{1}, x_{2}, \ldots, x_{q}$, das eine Lösung des Congruenzensystems (1) ist, den Werth 1 , für jedes andere den Werth 0 hat, und dass daher die Summe:

$$
\sum_{x_{1}, \cdots, x_{q}}^{0,1, \cdots, r-1} F\left(x_{1}|\cdots| x_{q}\right)
$$

den Werth $s$ besitzt. Man hat also für die Anzahl $s$ der Normallösungen des Congruenzensystems (1) den Ausdruck:

$$
s=\frac{1}{r^{p}} \sum_{\substack{x_{1}, \cdots, x_{q} \\ y_{1}, \cdots, y_{p}}}^{0,1, \cdots, r-1} e^{\frac{2 \pi i}{r}} \sum_{\mu=1}^{p} \sum_{v=1}^{q} a_{\mu \nu} x_{v} y_{\mu},
$$

wobei die angedeutete Summation so auszuführen ist, dass über jede der $p+q$ Grössen $x$ und $y$ unabhängig von den übrigen von 0 bis $r-1$ summirt wird. 
2.

Mit Hülfe des unter (2) für $s$ angegebenen Ausdrucks lässt sich nun sofort ein Satz beweisen. Nennt man nämlich die Anzahl der Normallösungen des zu (1) conjugirten Congruenzensystems:

$$
\sum_{\mu=1}^{p} a_{\mu \nu} x_{\mu}^{\prime} \equiv 0(\bmod . r) \quad(\nu=1,2, \ldots, q)
$$

$s^{\prime}$, so ist nach (2)

$$
s^{\prime}=\frac{1}{r^{2}} \sum_{\substack{x_{1}^{\prime}, \cdots, x_{p}^{\prime} \\ y_{1}^{\prime}, \cdots, y_{q}^{\prime}}}^{0,1, \cdots, r-1} e^{\frac{2 \pi i}{r} \sum_{\nu=1}^{q} \sum_{\mu=1}^{p} a_{\mu \nu} x_{\mu}^{\prime} y_{\nu}^{\prime}},
$$

und es ergiebt sich daraus, nachdem man für $\mu=1,2, \ldots, p$ und $\nu=1,2, \ldots, q x_{\mu}^{\prime}=y_{\mu}, y_{\nu}^{\prime}=x_{\nu}$ gesetzt hat, sofort durch Vergleichung mit (2) die Beziehung:

$$
r^{q} s^{\prime}=r^{p} s .
$$

Bezeichnet man also mit $s$ die Anzahl der Normallösungen des Congruenzensystems (1), mit $s$ die des conjugirten Congruenzensystems (3), so ist:

$$
\frac{r^{q}}{s}=\frac{r^{p}}{s^{\prime}}
$$

3.

Die in der Gleichung (4) stehenden Quotienten sind ganze Zahlen; es ist nämlich für ein Congruenzensystem (1) die Zahl s stets ein Theiler von $r^{q}$. Um dies einzusehen ordne man die sämmtlichen $r^{q}$ aus den Zahlen $0,1, \ldots, r-1$ zu bildenden Zahlensysteme $x_{1}, x_{2}, \ldots, x_{q}$ folgendermassen in Gruppen, wobei zur Abkürzung ein Zahlensystem $x_{1}, x_{2}, \ldots, x_{q}$ symbolisch mit $X$ bezeichnet und verschiedene solche Zahlensysteme durch obere Indices unterschieden werden mögen. Man betrachte die $p$ Linearformen:

$$
A_{\mu}=\sum_{\nu=1}^{q} a_{\mu \nu} x_{\nu} ; \quad(\mu=1,2, \ldots, p)
$$

lässt man darin an Stelle von $x_{1}, x_{2}, \ldots, x_{q}$ zunächst die $s$ Normallösungen des Congruenzensystems (1) treten, so wird

$$
A_{1} \equiv 0, A_{2} \equiv 0, \ldots, A_{p} \equiv 0 \text { (mod. } r \text { ); }
$$

diese $s$ Zahlensysteme $\boldsymbol{X}$ seien mit:

$$
X^{(1)}, X^{(2)}, \ldots, X^{(s)}
$$


bezeichnet. Entweder sind damit alle $r^{q}$ Zahlensysteme $X$ erschöpft, d. h. es ist $s=r$, dann ist der aufgestellte Satz bewiesen; oder es ist $s<r^{q}$, dann giebt es ausser diesen $s$ Zahlensystemen $X$ noch andere; ein beliebiges solches sei $X^{\prime}=\left(x_{1}^{\prime}, x_{2}^{\prime}, \ldots, x_{q}^{\prime}\right)$. Setzt man jetzt in den $p$ Linearformen (5) $x_{1}=x_{1}^{\prime}, x_{2}=x_{2}^{\prime}, \ldots, x_{q}=x_{q}^{\prime}$, so werden dieselben jedenfalls nicht alle $\equiv 0(\bmod , r)$; es möge

$$
A_{1} \equiv g_{1}^{\prime}, A_{2} \equiv g_{2}^{\prime}, \ldots, A_{p} \equiv g_{p}^{\prime}(\bmod . r)
$$

werden. Diese nämlichen Zahlen $g^{\prime}$ treten dann immer wieder auf, wenn man in den $p$ Formen (5) an Stelle von $x_{1}, x_{2}, \ldots, x_{q}$ jene $s$ Zahlensysteme einführt, welche aus dem Systeme $X^{\prime}$ durch Addition der Systeme $X^{(1)}, X^{(2)}, \ldots, X^{(s)}$ abgeleitet werden (wobei die auftretenden Zahlen $x^{\prime}+x$ auf ihre kleinsten positiven Reste nach dem Modul $r$ zu reduciren sind). Die so entstandenen $s$ Kahlensysteme seien mit

$$
X^{(s+1)}, X^{(s+2)}, \ldots, X^{(2 s)}
$$

bezeichnet; sie sind alle von einander und ron den Zahlensystemen $X^{(1)}, X^{(2)}, \ldots, X^{(s)}$ verschieden, zugleich sind es die sämmtlichen Zahlensysteme, welche $A_{1} \equiv g_{1}^{\prime}, A_{2} \equiv g_{2}^{\prime}, \ldots, A_{p} \equiv g_{p}^{\prime}$ machen. Entweder sind nun mit diesen zwei Reihen alle $r^{q}$ Zahlensysteme $X$ erschöpft, in welchem Falle $2 s=r^{q}$, also der Satz bewiesen ist, oder es giebt noch andere Zahlensysteme $X$, die in diesen zwei Reihen nicht vorkommen.

So fortfahrend kann man die sämmtlichen $r^{q}$ Zahlensysteme $X$ in Reihen von je $s$ anordnen in der Form:

$$
\begin{aligned}
& X^{(1)}, \quad X^{(2)}, \quad \ldots, X^{(s)} \text {; } \\
& X^{(s+1)}, \quad X^{(s+2)}, \quad \ldots, X^{(2 s)} \text {; } \\
& X^{(t-1 \cdot s+1)}, X^{(t-1 \cdot s+2)}, \ldots, X^{(t s)}
\end{aligned}
$$

wobei $t s=r^{q}$ ist, und erkennt daraus, dass die Anzahl $s$ der Normallösungen des Congruenzensystems (1) stets ein Theiler von $r^{q}$ ist. Die $s$ in einer Horizontalreihe stehenden Zahlensysteme sind dadurch charakterisirt, dass sie die $m$ Linearformen (5) den nämlichen Zahlen $g_{1}, g_{2}, \ldots, g_{p}$ congruent machen, und es sind zugleich die sämmtlichen Zahlensysteme, die dies thun.

Aus (6) schliesst man weiter sofort, dass ein System nicht homogener linearer Congruenzen:

$$
\sum_{\nu=1}^{q} a_{\mu \nu} x_{\nu} \equiv g_{i} \quad(\mu=1,2, \ldots, p)
$$


$g_{1}, g_{2}, \ldots, g_{p}$, für welche dieses Congruenzensystem Lösungen hat, durch die Formen (5) darstellbar, so ist die Anzahl der darstellbaren Zahlensysteme $t=\frac{r^{q}}{s} *$, und die Formel (4) lautet einfach dahin, dass durch $p$ Formen:

$$
A_{\mu}=\sum_{\nu=1}^{q} a_{\mu \nu} x_{\nu} \quad(\mu=1,2, \ldots, p)
$$

und durch die $q$ dazu conjugirten Formen:

$$
A_{\nu}^{\prime}=\sum_{\mu=1}^{p} a_{\mu \nu} x_{\mu}^{\prime} \quad(\nu=1,2, \ldots, q)
$$

stets gleich viele Zahlensysteme darstellbar sind $\left.{ }^{*}\right)$.

Ein Kriterium dafür, ob ein Congruenzensystem (7) Lösungen hat oder nicht, mit anderen Worten, ob ein Zahlensystem $g_{1}, g_{2}, \ldots, g_{p}$ durch gegebene Formen $A_{1}, A_{2}, \ldots, A_{p}$ darstellbar ist, wird in Art. 7 angegeben.

5.

Ein Fall kann sofort erledigt werden. Ist nämlich $p=q$ und die Determinante $\Sigma \pm a_{11} a_{22} \ldots a_{q q}= \pm 1$, so ist jedes Zahlensystem $g_{1}, g_{2}, \ldots, g_{p}$ durch die Formen $\overline{A_{1}}, A_{2}, \ldots, A_{q}$ darstellbar; es ist also in diesem Falle für jeden Werth des Moduls $r$ :

$$
t=r^{q}, s=1 \text {. }
$$

Von diesem Satze sei die folgende Anwendung gemacht. Lässt man in dem Gleichungensysteme:

$$
\sum_{\nu=1}^{q} a_{\mu \nu} x_{\nu}=x_{\mu}^{\prime}, \quad(\mu=1,2, \ldots, q)
$$

für welches die Determinante $\Sigma \pm a_{11} a_{22} \ldots a_{q q}$ den Werth \pm 1 hat, an Stelle des Systems der Grössen $x_{1}, x_{2}, \ldots, x_{q}$ der Reibe vach die sämmtlichen Variationen mit Wiederholung der Elemente $0,1, \ldots, r-1$ zur $q^{\text {ten }}$ Classe treten und denkt sich jedesmal die entstehenden Grössen $x_{1}^{\prime}, x_{2}{ }^{\prime}, \ldots, x_{q}^{\prime}$ auf ihre kleinsten positiven Reste nach dem Modul $r$ reducirt, so treten nach dem soeben Bemerkten an Stelle des Systems der $q$ Grössen $x_{1}^{\prime}, x_{2}^{\prime}, \ldots, x_{q}^{\prime}$ diese nämlichen Variationen nur in anderer Reihenfolge. Mit anderen Worten: wenn die Grössen $x_{1}, x_{2}, \ldots, x_{q}$ unabhängig von einander die Reihe der ganzen Zahlen $0,1, \ldots, r-1$ durchlanfen, so thun dies, mod. $r$ betrachtet, auch die 
Zahlen $x_{1}^{\prime}, x_{2}^{\prime}, \ldots, x_{q}^{\prime}$. Führt man daher in dem unter (2) angeschriebenen Ausdrucke für $s$ an Stelle der Summationsbuchstaben $x, y$ neue $x^{\prime}, y^{\prime}$ vermittelst der unimodularen linearen Substitutionen:

$$
\begin{array}{ll}
x_{\nu}=\sum_{\sigma=1}^{q} h_{\nu \sigma} x_{\sigma}^{\prime}, & y_{\mu}=\sum_{\rho=1}^{p} k_{\varrho \mu} y_{\varrho}^{\prime}, \\
(\nu=1,2, \ldots, q) & (\mu=1,2, \ldots, p)
\end{array}
$$

(wobei also $\Sigma \pm h_{11} h_{22} \ldots h_{q q}= \pm 1, \Sigma \pm k_{11} k_{22} \ldots k_{p p}= \pm 1$ ist) ein, so hat man auch über jeden dieser neuen Summationsbuchstaben unabhängig von den anderen von 0 bis $r-1$ zu summiren und erhält so, wenn man beachtet, dass:

$$
\sum_{\mu=1}^{p} \sum_{\nu=1}^{q} a_{\mu \nu} x_{\nu} y_{\mu}=\sum_{\varrho=1}^{p} \sum_{\sigma=1}^{q}\left(\sum_{\mu=1}^{p} \sum_{\nu=1}^{q} k_{\varrho \mu} a_{\mu \nu} h_{\nu \sigma}\right) x_{\sigma}^{\prime} y_{\varrho}^{\prime}
$$

wird, und zur Abkürzung:

$$
\sum_{\mu=1}^{p} \sum_{\nu=1}^{q} k_{\varrho \mu} a_{\mu \nu} h_{\nu \sigma}=b_{\varrho \sigma} \quad\left(\begin{array}{l}
\varrho=1,2, \ldots, p \\
\sigma=1,2, \ldots, q
\end{array}\right)
$$

setzt, für $s$ den neuen Ausdruck:

$$
s=\frac{1}{r^{p}} \sum_{\substack{x_{1}^{\prime}, \cdots, x_{q}^{\prime} \\ y_{1}^{\prime}, \cdots, y_{p}^{\prime}}}^{0,1, \cdots, r^{-1}} e^{\frac{2 \pi i}{r} \sum_{\rho=1}^{p} \sum_{\sigma=1}^{q}{ }_{0}{ }_{\rho} x_{\sigma^{\prime}} y_{\rho}^{\prime}},
$$

bei welchem man nun zum Zwecke der Berechnung voll $s$ über die ganzen Zahlen $h$ und $k$ innerhalb der Bedingungen

$$
\Sigma \pm h_{11} h_{22} \ldots h_{q q}= \pm 1, \quad \Sigma \pm k_{11} k_{22} \ldots k_{p p}= \pm 1
$$

frei verfügen darf.

6.

Der auf der rechten Seite der Gleichung (2) im Exponenten stehende Ausdruck:

$$
A=\sum_{\mu=1}^{p} \sum_{\nu=1}^{q} a_{\mu \nu} x_{\nu} y_{\mu}
$$

wird eine bilineare Form genannt. Verschwinden für die Matrix ihrer ganzzahligen Coefficienten $a_{\mu \nu}$ alle Determinanten $l+1^{\text {ten }}$ (und höheren) Grades, aber nicht alle Determinanten $l^{\text {ten }}$ Grades, so heisst $l$ der Rang der Form $A$. Man bilde, indem man unter $\lambda$ eine der Zahlen $1,2, \ldots, l$ versteht, alle Determinanten $\lambda^{\text {ten }}$ Grades und heisse $d_{\lambda}$ den grössten 
gemeinsamen Theiler derselben; die Quotienten $e_{\lambda}=\frac{d_{\lambda}}{d_{\lambda-1}}$, wobei im Falle $\lambda=1$ unter $d_{0}$ die Einheit zu verstehen ist, sind dann gleichfalls ganze Zahlen und heissen die Elementartheiler der Form $A$. Geht dann die Form $A$ durch unimodulare lineare Substitutionen (8) in die Form:

$$
B=\sum_{\rho=1}^{p} \sum_{\sigma=1}^{q} b_{\rho \sigma} x_{\sigma}^{\prime} y_{\varrho}^{\prime}
$$

über, wobei die Coefficienten $b_{\varrho \sigma}$ durch die Gleichungen (9) definirt sind, so ist jede Determinante $\lambda^{\text {ten }}$ Grades der $b$ eine homogene lineare Function der Determinanten $\lambda^{\text {ten }}$ Grades der $a$ und daher auch durch $d_{\lambda}$ theilbar; $d_{\lambda}$ ist aber zugleich der grösste gemeinsame Theiler aller Determinanten $\lambda^{\text {ten }}$ Grades der $b$, da auch die Form $B$ durch unimodulare lineare Substitutionen in die Form $A$ übergeführt werden kann, also auch jede Determinante $\lambda^{\text {ten }}$ Grades der $a$ eine homogene lineare Function der Determinanten $\lambda^{\text {ten }}$ Grades der $b$ ist. Nennt man daher zwei Formen wie $A$ und $B$ äquivalent, so sind für äquivalente Formen die Zahlen $d_{2}$ und daher auch die Elementartheiler $e_{2}$ die gleichen *).

Das in Art. 5 abgeleitete und in der Formel (10) niedergelegte Resultat kann jetzt dahin ausgesprochen werden, dass in dem Ausdrucke (2) für die Zahl $s$ die Form $A$ dureh jede beliebige dazu äquivalente ersetzt werden darf. Unter allen zu einer gegebenen Form $A$ äquivalenten Formen giebt es nun eine ausgezeichnete, die Normalform:

$$
E=\sum_{\lambda=1}^{l} e_{\lambda} x_{\lambda}^{\prime} y_{\lambda}^{\prime},
$$

deren Coefficienten $e_{1}, e_{2}, \ldots, e_{l}$ die vorher definirten Elementartheiler von $A$ sind $\left.{ }^{*}\right)$. Führt man aber diese Normalform $E$ an Stelle der Form $B$ in (10) ein, so erhält man für $s$ den Ausdruck:

$$
s=\frac{1}{r^{p}} \sum_{\substack{x_{i}^{\prime}, \cdots, x_{q}^{\prime} \\ y_{1}, \cdots, y_{p}^{\prime}}}^{0,1, \cdots, r-1} e^{\frac{2 \pi i}{r} \sum_{i=1}^{l} e_{\lambda} x_{\lambda} y_{\lambda}^{\prime}},
$$

der jetzt ohne Mühe ausgewerthet werden kann.

Zunächst kann die Summation nach den Grössen $x_{l+1}^{\prime}, \ldots, x_{q}^{\prime}$, $y_{i+1}^{\prime}, \ldots, y_{p}^{\prime}$, da von ihnen das allgemeine Glied der Summe unab- 
hängig ist, sofort ausgeführt werden, und weiter zerfällt dann die übrig bleibende $2 l$-fache Summe in das Product von $l$ Doppelsummen. Man erhält so für $s$ den Ausdruck:

$$
s=r^{q-2}-\prod_{\lambda=1}^{l}\left(\sum_{x_{2}^{\prime}, y_{\lambda}^{\prime}}^{0,1, \cdots, r-1} e^{\frac{2 \pi i}{r} e_{\lambda} x_{2}^{\prime} y_{\lambda}^{\prime}}\right) \text {. }
$$

Man bemerkt nun weiter, dass eine Summe:

$$
S_{\lambda}=\sum_{y_{\lambda}^{\prime}=0}^{r-1} e^{\frac{2 \pi i}{r} e_{\lambda}^{x_{\lambda}^{\prime} y_{\lambda}^{\prime}}}
$$

nur dann einen von Null verschiedenen Werth und zwar den Werth $r$ hat, wenn $e_{2} x_{2}^{\prime}$ durch $r$ ohne Rest theilbar ist; durchläuft $x_{2}^{\prime}$ aber die Zahlen $0,1, \ldots, r-1$, so kommt dies $s_{2}$-mal vor, wenn $s_{\lambda}$ den grössten gemeinsamen Theiler von $e_{\lambda}$ und $r$ bezeichnet, nämlich für die Werthe $x_{\lambda}^{\prime}=x \frac{r}{s_{\lambda}}\left(x=0,1, \ldots, s_{\lambda}-1\right) ;$ also ist:

$$
\sum_{x_{\lambda}^{\prime}, y_{\lambda}^{\prime}}^{0,1, \cdots, r-1} e^{\frac{2 \pi i}{r} e_{\lambda} x_{\lambda}^{\prime} y_{\lambda}^{\prime}}=\sum_{x_{2}^{\prime}=0}^{r-1} S_{\lambda}=s_{\lambda} \cdot r
$$

und daher endlich*):

$$
s=s_{1} s_{2} \ldots s_{i} \cdot r^{q-l}
$$

Man hat also das Hauptresultat: Die Anzahl s der Normallösungen des Congruenzensystems (1) beträgt $s=s_{1} s_{2} \ldots s_{2} r^{q-l}$, wenn $l$ der Rang der bilinearen Form $A, s_{\lambda}$ aber für $\lambda=1,2, \ldots, l$ der grösste gemeinsame Theiler von $r$ und dem $\lambda^{\text {ten }}$ Elementartheiler $e_{\lambda}$ von $A$ ist.

7.

Nachdem man jetzt im Stande ist, für jedes gegebene Congruenzensystem (1) die Anzahl seiner Normallösungen zu berechnen, kann auch die in Art. 4 aufgeworfene Frage beantwortet werden, welchen Bedingurgen ein Zahlensystem $g_{1}, g_{2}, \ldots, g_{p}$ genügen muss, damit das dort aufgestellte System nichthomogener linearer Congruenzen (7) Lösungen besitze.

Man betrachte die $p$ Linearformen:

$$
A_{\mu}=\sum_{\nu=1}^{q} a_{\mu \nu} x_{\nu} \quad(\mu=1,2, \ldots, p)
$$

*) Frobenius a. a. 0. p. 192 ; Smith a. a. 0. p. 399. 
und daneben die $p$ Linearformen:

$$
\overline{A_{\mu}}=\sum_{\nu=1}^{q} a_{\mu \nu} x_{\nu}-g_{\mu} x_{q+1} . \quad(\mu=1,2, \ldots, p)
$$

Jedes durch die Linearformen $A_{\mu}$ darstellbare Zahlensystem ist auch durch die Linearformen $\bar{A}_{\mu}$ darstellbar (man braucht nur $x_{q+1}=0 \mathrm{zu}$ setzen), es ist also $\bar{t} \geq t$, wenn die Anzahl der darstellbaren Zahlensysteme für die Linearformen $A_{\mu}$ wie früher mit $t$, für die Linearformen $\bar{A}_{\mu}$ mit $\bar{t}$ bezeichnet wird. Hat nun das Congruenzensystem (7) keine Lösung, so ist schon das Zahlensystem $g_{1}, g_{2}, \ldots, g_{p}$ selbst zwar durch die Linearformen $\bar{A}_{\mu}$ (man braucht nur $x_{1}=0, \ldots, x_{q}=0$, $x_{q+1}=r-1$ zu setzen), nicht aber durch die Linearformen $A_{\mu}$ darstellbar; es ist als in diesem Falle $\bar{t}>t$. Hat dagegen das Congruenzensystem (7) eine Lösung $x_{1}=\xi_{1}, x_{2}=\xi_{2}, \ldots, x_{q}=\xi_{q}$, so ist $\bmod . r$ :

$$
\sum_{\nu=1}^{q} a_{\mu \nu} x_{\nu}-g_{\mu} x_{q+1} \equiv \sum_{\nu=1}^{q} a_{\mu \nu}\left(x_{\nu}-\xi_{\nu} x_{q+1}\right) ; \quad(\mu=1,2, \ldots, p)
$$

es ist also jedes durch die Formen $\bar{A}_{\mu}$ darstellbare Zahlensystem auch durch die Formen $A_{\mu}$ darstellbar und sohin $\bar{t}=t$. Folglich ist $\bar{t}=t$ die nothwendige und hinreichende Bedingung dafür, dass das Congruenzensystem (7) eine Lösung besitze*). Da nun, wie früher angegeben, für ein Congruenzensystem (1) die Zahl $t$ mit der Anzahl $s$ der Normallösungen durch die Gleichung $t=\frac{r^{q}}{s}$ zusammenhängt, so zieht die Gleichung $\bar{t}=t$ für die entsprechenden Zahlen $\bar{s}$ und $s$ die Gleichung $\bar{s}=r s$ nach sich, und man kann daher das gefundene Resultat folgendermassen aussprechen. Damit das System der nichthomogenen linearen Congruenzen (7) Lösungen besitze, ist nothwendig und hinreichend, dass für das Congruenzensystem:

$$
\sum_{\nu=1}^{q} a_{\mu \nu} x_{\nu}-g_{\mu} x_{q+1} \equiv 0(\bmod . r) \quad(\mu=1,2, \ldots, p)
$$

die Anzahl der Normallösungen $\bar{s}=r s$ betrage, wenn $s$ die Anzahl der Normallösungen des Congruenzensystems (1) bezeichnet.

8.

Es sollen jetzt noch aus der Formel (13) einige Resultate abgeleitet werden, die bei Untersuchungen über Thetafunctionen Verwendung finden. 
Im Falle $l=p=q$ ergiebt sich unter der Annahme, dass der Modul $r$ ein Vielfaches der Determinante $\Delta=\Sigma \pm a_{11} a_{22} \ldots a_{q q}$ sei, folgendes einfache Resultat. $\mathrm{Da} e_{1} e_{2} \ldots e_{q}$ die Determinante der Normalform $E$ ist, diese aber mit der Determinante $\Delta$ der ursprünglichen Form bis aufs Vorzeichen übereinstimmt, so ist $\nabla=e_{1} e_{2} \ldots e_{q}$, wenn $\nabla$ den absoluten Werth von $\Delta$ bezeichnet. Ist nun $r=g \nabla$, wo $g$ eine positive ganze $\mathrm{Zahl}$ ist, so ist für $\lambda=1,2, \ldots, q: s_{\lambda}=e_{\lambda}$ und daher $s=e_{1} e_{2} \ldots e_{q}=\nabla$. Man hat also den Satz, dass, wenn nur die Determinante $\Delta=\Sigma \pm a_{11} a_{22} \ldots a_{q q}$ von $N u l l$ verschieden ist, die Anzahl der Normallösungen des Congruenzensystems:

$$
\sum_{\nu=1}^{q} a_{\mu \nu} x_{\nu} \equiv 0(\bmod . g \nabla) \quad(\mu=1,2, \ldots, q)
$$

für jede ganze Zahl $g$ stets gleich dem absoluten Werthe $\nabla$ der Determinante $\Delta$ ist.

\section{9.}

Ist wieder $l=p=q$, der Modul $r$ aber relativ prim zu $\Delta$, also auch wegen $\nabla=e_{1} e_{2} \ldots e_{q}$ relativ prim zu jedem Elementartheiler $e_{\lambda}$, so sind alle Grössen $s_{\lambda}$ und daher auch $s=1$. Es hat also das Congruenzensystem:

$$
\left.\sum_{v=1}^{q} a_{\mu \nu} x_{v} \equiv 0 \text { (mod. } r\right), \quad(\mu=1,2, \ldots, q)
$$

bei welchem wieder die Determinante $\Delta=\Sigma \pm a_{11} a_{22} \ldots a_{q q} \neq 0$ sei, in dem Falle, dass der Modul $r$ relativ prim zu $\Delta$ ist, nur eine einzige Normallösung, nämlich $\left.x_{1}=0, x_{2}=0, \ldots, x_{q}=0^{*}\right)$.

10.

Man betrachte ferner das Congruenzensystem:

$$
\sum_{\mu=1}^{q} \alpha_{\mu \nu} x_{\mu} \equiv 0(\bmod . \nabla), \quad(\nu=1,2, \ldots, q)
$$

bei welchem $a_{\mu \nu}$ die Adjuncte von $a_{\mu \nu}$ in der Determinante:

$$
\Delta=\Sigma \pm a_{11} a_{22} \ldots a_{q q}
$$

bezeichne. Bekanntlich ist jede Unterdeterminante $\lambda^{\text {ten }}$ Grades der $\alpha$ dem $\Delta^{2-1}$-fachen der zugehörigen Adjuncte $q-\lambda^{\text {ten }}$ Grades der $a$ gleich; bezeichnet man also mit $\delta_{\lambda}$ den grössten gemeinsamen Theiler 
der Determinanten $\lambda^{\text {ten }}$ Grades der $\alpha$, so ist $\delta_{\lambda}=\nabla^{\lambda-1} \cdot d_{q-\lambda}$, und es hat daher für die bilineare Form:

$$
\sum_{\mu=1}^{q} \sum_{\nu=1}^{q} \alpha_{\mu \nu} x_{\nu} y_{\mu}
$$

$\operatorname{der} \lambda^{\text {te }}$ Elementartheiler $\varepsilon_{\lambda}$ den Werth:

$$
\varepsilon_{\lambda}=\nabla \frac{d_{q-\lambda}}{d_{q-2+1}}=\frac{\nabla}{e_{q-\lambda+1}} .
$$

Es ist also weiter mit Rücksicht auf das Congruenzensystem (17) der grösste gemeinsame Theiler $\sigma_{\lambda}$ von $\varepsilon_{2}$ und dem Modul $\nabla \varepsilon_{\lambda}$ selbst und daher endlich die Anzahl $\sigma$ der Normallösungen des Congruenzensystems (17):

$$
\sigma=\sigma_{1} \sigma_{2} \ldots \sigma_{q}=\varepsilon_{1} \varepsilon_{2} \ldots \varepsilon_{q}=\frac{\nabla^{q}}{e_{q} e_{q-1} \ldots e_{1}}=\nabla^{q-1} .
$$

11.

Man betrachte endlich das im ersten Abschnitte unter (12) auftretende Congruenzensystem:

$$
r \sum_{\mu=1}^{q} \alpha_{\mu \nu} x_{\mu} \equiv 0(\bmod . \nabla) . \quad(\nu=1,2, \ldots, q)
$$

Nennt man bei diesem den grössten gemeinsamen Theiler der Determinanten $\lambda^{\text {ten }}$ Grades seiner Coefficienten $\delta_{\lambda}^{\prime}$ und den $\lambda^{\text {ten }}$ Elementartheiler der zugehörigen bilinearen Form $\varepsilon_{2}^{\prime}$, so ist:

$$
\delta_{\lambda}^{\prime}=r^{\lambda} \nabla^{\lambda-1} a_{q-\lambda}, \quad \varepsilon_{\lambda}^{\prime}=\frac{r \nabla}{e_{q-\lambda+1}} .
$$

Folglich ist der grösste gemeinsame Theiler $\sigma_{2}^{\prime}$ von $\varepsilon_{2}^{\prime}$ und dem Modul $\nabla$ :

$$
\sigma_{\lambda}^{\prime}=\frac{s_{q-\lambda+1} \nabla}{e_{q-\lambda+1}}
$$

wenn wie früher mit $s_{q-\lambda+1}$ der grösste gemeinsame Theiler von $e_{q-\lambda+1}$ und $r$ bezeichnet wird, and es ist also endlich die Anzahl $\sigma^{*}$ der Normallösungen des Congruenzensystems (19):

$$
\sigma^{\prime}=\sigma_{1}^{\prime} \sigma_{2}^{\prime} \ldots \sigma_{q}^{\prime}=\frac{s_{q} s_{q-1} \ldots s_{1} \nabla^{q}}{e_{q} e_{q-1} \ldots e_{1}}=s \cdot \nabla^{q-1},
$$

wenn wie immer mit $s$ die nach (13) zu berechnende Anzahl der Normallösungen des Congruenzensystems (1) bezeichnet wird. 
Führt man den unter (20) gefundenen Werth an Stelle von $\sigma^{\prime}$ in die Endformel (18) des ersten Abschnittes ein, so stellt sich die durch die lineare Substitution:

$$
r m_{\mu}=\sum_{\nu=1}^{q} a_{\mu \nu} n_{\nu}, \quad(\mu=1,2, \ldots, q)
$$

bei der $r$ eine positive ganze Zahl, die $a_{\mu \nu}$ ganze Zahlen mit nicht verschwindender Determinante bezeichnen, bewirkte Umformung der $q$-fach unenalichen Reihe:

$$
W=\sum_{m_{1}, \cdots, m_{q}}^{-\infty, \ldots,+\infty} f\left(m_{1}|\ldots| m_{q}\right)
$$

dar in der Gleichung:

$$
\begin{gathered}
W=\frac{1}{r^{q} \nabla^{q-1} s} \sum_{\rho_{1}, \cdots, \varrho_{q}}^{0,1, \ldots, \nabla-1} \sum_{\sigma_{1}, \ldots, \sigma_{q}}^{0,1, \ldots, r-1} \\
\left(\sum_{n_{2}, \cdots, n_{q}}^{-\infty, \ldots,+\infty} e^{\frac{2 \pi i}{r} \sum_{\nu=1}^{q}\left(n_{\nu}+\frac{\overline{\bar{\rho}_{v}}}{\Delta}\right){\overline{\sigma_{\nu}}}_{\nu}} g\left(n_{1}+\frac{\overline{\bar{\rho}_{1}}}{\Delta}|\ldots| n_{q}+\frac{\overline{\bar{\rho}}_{q}}{\Delta}\right)\right) .
\end{gathered}
$$

Dabei ist die Function $g\left(n_{1}|\ldots| n_{q}\right)$ durch die Gleichung:

$$
f\left(m_{1}|\ldots| m_{q}\right)=g\left(n_{1}|\ldots| n_{q}\right)
$$

definirt; es ist ferner zur Abkürzung:

$$
\overline{\bar{\varrho}}_{v}=r \sum_{\mu=1}^{q} \alpha_{\mu \nu} \varrho_{\mu}, \quad \bar{\sigma}_{\nu}=\sum_{\mu=1}^{q} a_{\mu \nu} \sigma_{\mu} \quad(\nu=1,2, \ldots, q)
$$

gesetzt; es bezeichnet $\Delta$ den Werth, $\nabla$ den absoluten Werth der Determinante $\Sigma \pm a_{11} a_{22} \ldots a_{q q}$ und $\alpha_{\mu \nu}$ die Adjuncte von $a_{\mu \nu}$ in dieser Determinante, und es ist endlich unter $s$ die nach der Formel (13) zu berechnende Anzahl der Normallösungen des Congruenzensystems:

$$
\sum_{\nu=1}^{q} a_{\mu \nu} x_{\nu} \equiv 0(\bmod . r) \quad(\mu=1,2, \ldots, q)
$$

verstanden. 


\section{Dritter Abschnitt.}

\section{Von den Umformungen eines Thetaproductes durch lineare Substitution der Summationsbuchstaben.}

1.

Die allgemeinen Substitutionen $S$.

Gegeben sei das Thetaproduct:

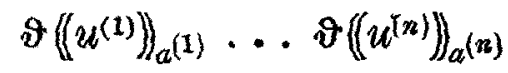

$$
\begin{aligned}
& -\sum_{[m]}^{\infty} \ldots+\cdots e^{\rho} \sum^{n}\left(\sum_{\mu=1}^{p} \sum_{\mu^{\prime}=1}^{p} a_{\mu \mu^{\prime}}^{(\rho)} m_{\mu}^{(\rho)} m_{\mu}^{(\rho)}+2 \sum_{\mu=1}^{p} m_{\mu}^{(\rho)} u_{\mu}^{(\varrho)}\right),
\end{aligned}
$$

wobei unter dem Summenzeichen das System der $n p$ Summationsbuchstaben $m_{\mu}^{(\rho)}\left(\begin{array}{l}\rho=1,2, \ldots, n \\ \mu=1,2, \ldots, p\end{array}\right)$ abgekürat mit $[m]$ bezeichnet und die angedeutete Summation so auszuführen ist, dass nach jedem dieser $n p$ Summationsbuchstaben unabhängig von den anderen von $-\infty$ bis $+\infty$ summirt werden soll. Die auf der rechten Seite dieser Gleichung stehende $n p$-fach unendliche Reihe soll dadureh umgeformt werden, dass man an Stelle der Summationsbuchstaben $m$ neue Summationsbuchstaben $n$ einführt mit Hülfe der Substitution:

$$
m_{\mu}^{(\rho)}=\sum_{\sigma=1}^{n} \sum_{\nu=1}^{p} r_{\mu \nu}^{(\rho)} n_{\nu}^{(\sigma)}, \quad\left(\begin{array}{l}
\rho=1,2, \ldots, n \\
\mu=1,2, \ldots, p
\end{array}\right)
$$

bei der die $r_{\mu \nu}^{(0)}(n p)^{2}$ rationale Zahlen mit nicht verschwindender Determinante bezeichnen, und es sollen dabei die Zablen $r$ in allgemeinster Weise so bestimmt werden, dass die nach Ausführung der Substitution $S$ auftretende $n p$-fach unendliche Reihe ein Aggregat von Producten von $n$ Thetafunctionen von je $p$ Veränderlichen wird.

Unter Anwendung der Substitution $S$ wird nun:

$$
\begin{gathered}
\sum_{\rho=1}^{n} \sum_{\mu=1}^{p} \sum_{\mu^{\prime}=1}^{p} a_{\mu \mu^{\prime}}^{(\rho)} m_{\mu}^{(\rho)} m_{\mu^{\prime}}^{(\rho)} \\
=\sum_{\sigma=1}^{n} \sum_{\sigma^{\prime}=1}^{n} \sum_{\nu=1}^{p} \sum_{\nu^{\prime}=1}^{p}\left(\sum_{\rho=1}^{n} \sum_{\mu=1}^{p} \sum_{\mu^{\prime}=1}^{p} a_{\mu \mu^{\prime}}^{(\rho)} r_{\mu \nu}^{(\rho)} r_{\mu^{\prime} \nu^{\prime}}^{\left(\rho \sigma^{\prime}\right)}\right) n_{\nu}^{(\sigma)} n_{\nu^{\prime}}^{\left(\sigma^{\prime}\right)} ;
\end{gathered}
$$

soll also die neue $n p$-fach unendliche Reibe wieder Producte von $n$ Thetafunctionen von je $p$ Veränderlichen liefern, so müssen die Coefficienten $r_{\mu \nu}^{(\rho \sigma)}$ ron $S$ den $\frac{1}{2}(n-1) n p^{2}$ Bedingungen: 


$$
\sum_{\varrho=1}^{n} \sum_{\mu=1}^{p} \sum_{\mu^{\prime}=1}^{p} a_{\mu \mu^{\prime}}^{(\rho)} r_{\mu \nu}^{(\rho \sigma)} r_{\mu^{\prime} \nu^{\prime}}^{\left(\rho^{\prime}\right)}=0 \quad\left(\begin{array}{l}
\sigma, \sigma^{\prime}=1,2, \ldots, n ; \sigma<\sigma^{\prime} \\
\nu, \nu^{\prime}=1,2, \ldots, p
\end{array}\right)
$$

genügen. Sind diese Bedingungen erfüllt, und setzt man dann:

und :

$$
\sum_{\rho=1}^{n} \sum_{\mu=1}^{p} \sum_{\mu^{\prime}=1}^{p} a_{\mu \mu^{\prime}}^{(\rho)} r_{\mu \nu}^{(\rho \sigma)} r_{\mu^{\prime} \nu^{\prime}}^{(\rho \sigma)}=b_{y \nu^{\prime}}^{(\sigma)} \quad\left(\begin{array}{r}
\sigma=1,2, \ldots, n \\
\nu, \nu^{\prime}=1,2, \ldots, p
\end{array}\right)
$$

$$
\sum_{\rho=1}^{n} \sum_{\mu=1}^{p} r_{\mu \nu}^{(\rho \sigma)} u_{\mu}^{(\rho)}=v_{\nu}^{(\sigma)}
$$$$
\left(\begin{array}{l}
\sigma=1,2, \ldots, n \\
\nu=1,2, \ldots, p
\end{array}\right)
$$

so geht, wie die am Ende des vorigen Abschnittes angeschriebene Hauptformel $(\boldsymbol{H})$ unmittelbar erkennen lässt, das vorgelegte Thetaproduct mit den Modulen $a_{\mu \mu^{\prime}}^{(\rho)}\left(\begin{array}{c}\rho=1,2, \ldots, n \\ \mu, \mu^{\prime}=1,2, \ldots, p\end{array}\right)$ und den Argumenten $u_{\mu}^{(\rho)}\left(\begin{array}{l}\rho=1,2, \ldots, n \\ \mu=1,2, \ldots, p\end{array}\right)$ in ein Aggregat von Thetaproducten mit den Modulen $b_{\nu \nu^{\prime}}^{(\sigma)}\left(\begin{array}{c}\sigma=1,2, \ldots, n \\ \nu, \nu^{\prime}=1,2, \ldots, p\end{array}\right)$ and den Argumenten $v_{\nu}^{(\sigma)}\left(\begin{array}{c}\sigma=1,2, \ldots, n \\ \nu=1,2, \ldots, p\end{array}\right)$ über. Die Gleichungen (2) stellen also die nothwendigen und hinreichenden Bedingungen dar, denen die Coefficienten der Substitution $S$ zu genügen haben*).

*) Zur Convergenz der neuen Thetareihen bedarf es keiner weiteren Voraussetzungen. Bėzeichnet man nämlich, wie üblich, den reellen Theil von $a_{\mu \mu}^{(\rho)}$, mit $a_{\mu \mu^{\prime}}^{(\rho)}$, den reellen Theil von $b_{\nu_{\nu^{\prime}}}^{(\sigma)}$ mit $b_{\nu v^{\prime}}^{(\sigma)}$, so erhält man wegen der Gleichungen (3):

$$
\sum_{\nu=1}^{n} \sum_{i=1}^{p} b_{v v^{\prime}}^{(\sigma)} y_{\nu} y_{v^{\prime}}=\sum_{\varrho=1}^{n}\left(\sum_{\mu=1}^{p} \sum_{\mu^{\prime}=1}^{p} a_{\mu}^{\prime(\rho)} x_{\mu}^{(\rho)} x_{\mu^{\prime}}^{(\varrho)}\right)
$$

wenn man zur Abkürzung:

$$
\sum_{\nu=1}^{p} r_{\mu \nu}^{(\rho \sigma)} y_{\nu}=x_{\mu}^{(\varrho)} \quad\left(\begin{array}{l}
\varrho=1,2, \ldots, n \\
\mu=1,2, \ldots, p
\end{array}\right)
$$

setzt, und erkennt daraus sofort, dass die auf der linken Seite stehende quadratische Form:

$$
\sum_{\nu=1}^{p} \sum_{\nu^{\prime}=1}^{p} b_{\nu \nu^{\prime}}^{(\sigma)} y_{\nu} y_{\nu^{\prime}}
$$

eine negative ist, sobald die $n$ auf der rechten Seite vorkommenden Formen:

$$
\sum_{\mu=1}^{p} \sum_{\mu^{\prime}=1}^{p} a_{\mu \mu^{\prime}}^{(\rho)} x_{\mu}^{(\rho)} x_{\mu^{\prime}}^{(\rho)} \quad(\rho=1,2, \ldots, n)
$$

es sind; d. h. dass jede der $n$ neuen Thetareihen convergirt, wenn die $n$ gegebenen es thun. 


\section{2.}

Die Substitutionen $E$.

Von den Substitutionen $S$ wurden von Herrn Prym und mir nur zwei specielle Arten untersucht. Die erste Art ist dadurch charakterisirt, dass von den Coefficienten $r_{\mu \nu}^{(\rho \sigma)}$ nur jene von Null verschieden sind, bei denen $\rho=\sigma$ ist. Eine solche Substitution wird also durch ein Gleichungensystem von der Form:

$$
m_{\mu}^{(\varrho)}=\sum_{\nu=1}^{p} r_{\mu \nu}^{(\varrho)} n_{\nu}^{(\varrho)} \quad\left(\begin{array}{l}
\varrho=1,2, \ldots, n \\
\mu=1,2, \ldots, p
\end{array}\right)
$$

definirt. Bedingungen für die Coefficienten $r_{\mu \nu}^{(\rho)}$ treten dabei, ausser dass die $n$ Determinanten $\Sigma \pm r_{11}^{(\varrho)} r_{22}^{(\ell)} \ldots r_{p p}^{(\varrho)}(\varrho=1,2, \ldots, n)$ sämmtlich von Null verschieden sein müssen, nicht auf, da durch die gemachte Annahme, wonach $r_{\mu \nu}^{(\varrho \sigma)}=0$ ist, sobald $\varrho \gtrless \sigma$ ist, die Bedingungen (2) bereits erfüllt sind. Die Substitution $E$ ist eine zerfallende; sie zerfällt in $n$ getrennte Substitutionen, von denen die $i^{\text {te }}$ :

$$
m_{\mu}^{(i)}=\sum_{\nu=1}^{p} r_{\mu \nu}^{(i)} n_{\nu}^{(i)} \quad(\mu=1,2, \ldots, p)
$$

die $i^{\text {te }}$ Thetafunction $\vartheta\left(\left(u^{(i)}\right)\right)_{a}(i)$ allein betrifft, und es vollzieht sich daher die durch die Substitution $E$ bewirkte Unformung des Thetaproductes (1) in der Weise, dass jede der $n$ Thetafunctionen für sich transformirt und die $n$ so entstandenen Gleichungen schliesslich mit einander multiplicirt werden. Es genügt also die Umformung einer einzelnen Thetafunction durch eine Substitution von der Form (5) anzugeben. Indem man zu dem Ende die $p^{2}$ rationalen Zahlen $r_{\mu \nu}^{(i)}$ auf gemeinsamen Nenner $r$ bringt und der Einfachheit halber den Index $i$ unterdrückt, entsteht die Aufgabe, die $p$-fach unendliche Thetareihe:

$$
\vartheta((u))_{\alpha}=\sum_{m_{1}, \cdots, m_{p}}^{-\infty, \cdots,+\infty} \sum^{p=1} \sum_{\mu^{\prime}=1}^{p} a_{\mu \mu^{\prime} m_{\mu} m_{\mu^{\prime}}+2} \sum_{\mu=1}^{p} m_{\mu} u_{\mu}
$$

durch die Substitution:

$$
r m_{\mu}=\sum_{v=1}^{p} e_{\mu \nu} n_{v}, \quad(\mu=1,2, \ldots, p)
$$

bei der $r$ eine positive ganze Zahl, die $e_{\mu \nu}$ ganze Zahlen mit nicht verschwindender Determinante bezeichnen, umzuformen. Diese Umformung erhält man aber aus der am Ende des vorigen Abschnittes 


$$
\begin{aligned}
& r^{p} \nabla^{p-1} s \vartheta((u))_{a}
\end{aligned}
$$

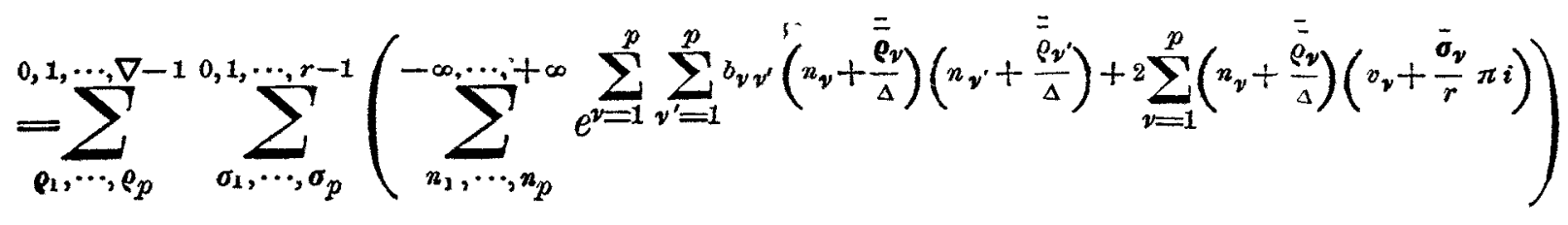

oder, indem man die auf der rechten Seite hinter den beiden ersten Summenzeichen stehende $p$-fach unendliche Reihe durch die mit ihr identische Thetafunction ersetzt, in der Thetaformel:

$$
r^{p} \nabla^{p-1} s \boldsymbol{v}((u))_{a}=\sum_{\rho_{1}, \cdots, \rho_{p}}^{0,1, \cdots, \nabla-1} \sum_{\sigma_{1}, \cdots, \sigma_{p}}^{0,1, \ldots, \dot{r}^{-1}} \vartheta\left[\begin{array}{c}
\overline{\bar{\rho}} \\
\bar{\Delta} \\
\frac{\bar{\sigma}}{r}
\end{array}\right]\left((v)_{b} .\right.
$$

Dabei sind die Grössen $v, b$ mit den Grössen $u, a$ durch die Gleichungen :

$$
\begin{gathered}
v_{\nu}=\frac{1}{r} \sum_{\mu=1^{\prime}}^{p} e_{\nu \mu} u_{\mu}, \quad b_{\nu \nu^{\prime}}=\frac{1}{r^{2}} \sum_{\mu=1}^{p} \sum_{\mu^{\prime}=1}^{p} e_{\nu \mu} e_{\nu^{\prime} \mu^{\prime}} a_{\mu \mu^{\prime}} \\
\left(\nu, \nu^{\prime}=1,2, \ldots, p\right)
\end{gathered}
$$

verknüpft; es ist ferner zur Abkürzung:

$$
\overline{\bar{\varrho}}_{\nu}=r \sum_{\mu=1}^{p} \varepsilon_{\nu \mu} \varrho_{\mu}, \quad \bar{\sigma}_{\nu}=\sum_{\mu=1}^{p} e_{\nu \mu} \sigma_{\mu} \quad(\nu=1,2, \ldots p)
$$

gesetzt; es bezeichnet weiter $\Delta$ den Werth, $\nabla$ den absoluten Werth der Determinante $\Sigma \pm e_{11} e_{22} \ldots e_{p p}$ und $\varepsilon_{\mu \nu}$ die Adjuncte von $e_{\mu \nu}$ in dieser Determinante, und es ist endlich unter $s$ die nach Formel (13) des zweiten Abschnittes zu berechnende Anzahl der Normallösungen des Congruenzensystems:

$$
\sum_{\nu=1}^{p} e_{\mu \nu} x_{\nu} \equiv 0(\bmod . r)
$$

$$
(\mu=1,2, \ldots, p)
$$

verstanden.

Man wird noch bemerken, dass die Substitutionen $E$ eine Gruppe bilden, dass also irgend zwei oder mehrere $E$-Substitutionen sich wieder zu einer $\boldsymbol{E}$-Substitution zusammensetzen, und dass die inverse einer $E$-Substitution selbst wieder eine $E$-Substitution ist; endlich aber, dass eine Substitution $E$ die einzig mögliche, zur Umformung eines Thetaproductes (1) anwendbare Substitution $S$ ist, wenn die $n$ gegebenen Thetafunctionen allgemeine und ihre Modulen vollständig von einander unabhängig sind; denn bestehen zwischen den Modulen

$$
a_{\mu \mu^{\prime}}^{(\varrho)}\left(\begin{array}{r}
\varrho \\
\mu, \mu^{\prime}=1,2, \ldots, n \\
=1,2, \ldots, p
\end{array}\right)
$$


keinerlei Beziehungen, so können, da dann für alle Werthe der Indices:

$$
r_{\mu \nu}^{(\rho \sigma)} r_{\mu \nu}^{\left(\rho \sigma^{\prime}\right)}=0
$$

sein muss, sobald $\sigma \gtrless \sigma^{\prime}$ ist, die Relationen (2) nur durch die Coefficienten einer Substitution $E$ erfüllt werden.

3.

Die Substitutionen $D$.

Die zweite von Herrn Prym und mir untersuchte specielle Art von Substitutionen $S$ ist dadurch charakterisirt, dass von den Coefficienten $r_{\mu \nu}^{(\varrho \sigma)}$ nur jene von Null verschieden sind, für welche $\mu=\nu$ ist. Eine solche Substitution wird also durch ein Gleichungensystem von der Form:

$$
m_{\mu}^{(\varrho)}=\sum_{\sigma=1}^{n} r_{\mu}^{(\rho)} n_{\mu}^{(\sigma)} \quad\left(\begin{array}{l}
\varrho=1,2, \ldots, n \\
\mu=1,2, \ldots, p
\end{array}\right)
$$

definirt. Auch eine Substitution $D$ ist eine zerfallende; sie zerfällt in $p$ Substitutionen, von denen die $k^{\text {te }}$ :

$$
m_{k}^{(\varrho)}=\sum_{\sigma=1}^{n} r_{k}^{(\varrho \sigma)} n_{k}^{(\sigma)} \quad(\varrho=1,2, \ldots, n)
$$

ist, von denen aber jede alle $n$ Thetafunctionen betriffit, und die daher hier nicht gesondert betrachtet werden können. Die Coefficienten der Substitution $D$, für welche die $p$ den einzelnen Substitutionen (12) zugehörigen Determinanten $\Sigma \pm r_{k}^{(11)} r_{k}^{(22)} \ldots r_{k}^{(n n)}$ sämmtlich von Null verschieden sein müssen, sind ausserdem an die $\frac{1}{2}(n-1) n p^{2}$ aus (2) folgenden Bedingungen:

$$
\sum_{\rho=1}^{n} a_{\mu \mu^{\prime}}^{(\rho)} r_{\mu}^{(\rho \sigma)} r_{\mu^{\prime}}^{\left(\rho \sigma^{\prime}\right)}=0 \quad\left(\begin{array}{l}
\sigma, \sigma^{\prime}=1,2, \ldots, n ; \sigma<\sigma^{\prime} \\
\mu, \mu^{\prime}=1,2, \ldots, p
\end{array}\right)
$$

geknüpft, während die Moduln $b_{\mu \mu^{\prime}}^{(\sigma)}$ und die Argumente $v_{\mu}^{(\sigma)}$ der neuen Thetafunctionen durch die aus (3) und (4) folgenden Gleichungen:

bestimmt sind.

$$
\begin{gathered}
b_{\mu \mu^{\prime}}^{(\sigma)}=\sum_{\rho=1}^{n} a_{\mu \mu^{\prime}}^{(\rho)} r_{\mu}^{(\rho)} r_{\mu^{\prime}}^{(\rho \sigma)}, \quad v_{\mu}^{(\sigma)}=\sum_{\varrho=1}^{n} r_{\mu}^{(\rho)} u_{\mu}^{(\rho)} \\
\left(\begin{array}{c}
\sigma=1,2, \ldots, n \\
\mu, \mu^{\prime}=1,2, \ldots, p
\end{array}\right)
\end{gathered}
$$

Die gefundenen Bedingungen (13) sird aber nicht nur Bedingungen für die Coefficienten $r_{\mu}^{(\varrho \sigma)}$ der Substitution $D$ sondern auch für die 
Modulen $a_{\mu \mu^{\prime}}^{(\rho)}$ des gegebenen Thetaproductes. Damit nämlich die Gleichungen (13) durch rationale Zahlen $r_{\mu}^{(\rho \sigma)}$ von nicht verschwindender Determinante erfüllt werden können, müssen die Modulen $a_{\mu \mu^{\prime}}^{(\rho)}$ in gewissem Zusammenhange mit einander stehen, und es erfordert demnach die Anwendung einer D-Substitution von vorneherein eine besondere Beschaffenheit des gegebenen Thetaproductes. Um diese aufzudecken leite man aus dem System ron Relationen:

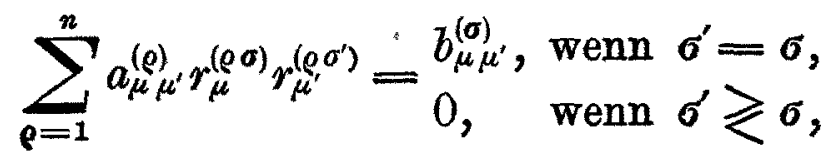

$$
\left(\begin{array}{l}
\sigma, \sigma^{\prime}=1,2, \ldots, n \\
\mu, \mu^{\prime}=1,2, \ldots, p
\end{array}\right)
$$

ein System einfacherer Relationen ab. Zu dem Ende bezeichne man mit $s_{\mu}^{(\rho \sigma)}$ die durch den Werth der Determinante $\Sigma \pm r_{\mu}^{(11)} r_{\mu}^{(22)} \ldots r_{\mu}^{(n n)}$ dividirte Adjuncte $n-1^{\text {ten }}$ Grades von $r_{\mu}^{(\rho \sigma)}$ in dieser Determinante, multiplicire, indem man unter $\varrho^{\prime}$ irgend eine Zahl aus der Reihe $1,2, \ldots, n$ versteht, linke und rechte Seite der Gleichung (15) mit $s_{\mu}^{\left(\rho^{\prime} \sigma^{\prime}\right)}$ und summire nach $\sigma^{\prime}$ von 1 bis $n$; man erhält dann, wenn man nach geschehener Summation schliesslich noch den Accent bei $\varrho^{\prime}$ unterdrückt, die Gleichung:

$$
r_{\mu}^{(\varrho \sigma)} a_{\mu \mu^{\prime}}^{(\varrho)}=s_{\mu^{\prime}}^{(\varrho \sigma)} b_{\mu \mu^{\prime}}^{(\sigma)}
$$

$$
\left(\begin{array}{l}
\varrho, \sigma=1,2, \ldots, n \\
\mu, \mu^{\prime}=1,2, \ldots, p
\end{array}\right)
$$

Aus den Gleichungen (16) folgt nun zunächst, dass, wenn von den $p$ Gleichungssystemen (12), aus denen die Substitution $D$ besteht, eines, etwa das $k^{\mathrm{te}}$ in Folge des Verschwindens gewisser seiner Coefficienten $r_{k}^{(\varrho \sigma)}$ in der Weise zerfällt, dass $m$ von den $n$ Zahlen $n_{k}^{(1)}, n_{k}^{(2)}, \ldots, n_{k}^{(n)}$ nur in $m$ seiner $n$ Gleichungen, die übrigen $n-m$ Zahlen $n_{k}$ nur in den $n-m$ übrigen Gleichungen vorkommen, dann die sämmtlichen $p$ Gleichungensysteme in derselben Weise zerfallen. Schliesst man aber diesen Fall, in der Erwägung, dass dann die verlangte Umformung des gegebenen Productes von $n$ Thetafunctionen sich in der Weise vollzieht, dass das Product von $m$ dieser Functionen und ebenso das Product der $n-m$ übrigen jedes für sich transformirt and die beiden so entstandenen Gleichungen mit einander multiplicirt werden, aus, so folgert man aus den Gleichungen (16) ohne Mühe, dass der Quotient von irgend zwei der $2 n$ Grössen

$$
a_{\mu \mu^{\prime}}^{(1)}, \ldots, a_{\mu \mu^{\prime}}^{(n)}, b_{\mu \mu^{\prime}}^{(1)}, \ldots, b_{\mu \mu^{\prime}}^{(n)}
$$

eine rationale $\mathrm{Zahl}$, dass also:

$$
a_{\mu \mu^{\prime}}^{(\varrho)}=h_{\mu \mu^{\prime}}^{(\varrho)} a_{\mu \mu^{\prime}}, \quad b_{\mu \mu^{\prime}}^{(\sigma)}=k_{\mu \mu^{\prime}}^{(\sigma)} a_{\mu \mu^{\prime}} \quad\left(\begin{array}{l}
\varrho, \sigma=1,2, \ldots, n \\
\mu, \mu^{\prime}=1,2, \ldots, p
\end{array}\right)
$$

ist, wo die $h, k$ ganze Zahlen bezeichnen. Beachtet man aber weiter die aus (16) folgende Gleichung: 


$$
\frac{a_{\mu \mu^{\prime}}^{(\varrho)} a_{\mu^{\prime} \mu^{\prime \prime}}^{(\varrho)} a_{\mu^{\prime \prime} \mu}^{(\varrho)}}{a_{\mu \mu}^{(\varrho)} a_{\mu^{\prime} \mu^{\prime}}^{(\varrho)} a_{\mu^{\prime \prime} \iota^{\prime \prime}}^{(\rho)}}=\frac{b_{\mu \mu^{\prime}}^{(\sigma)} b_{\mu^{\prime} \mu^{\prime \prime}}^{(\sigma)} b_{\mu^{\prime \prime} \mu}^{(\sigma)}}{b_{\mu \mu}^{(\sigma)} b_{\mu^{\prime} \mu^{\prime}}^{(\sigma)} b_{\mu^{\prime \prime} \mu^{\prime \prime}}^{(\sigma)}}, \quad\left(\begin{array}{r}
\rho, \sigma=1,2, \ldots, n \\
\mu, \mu^{\prime}, \mu^{\prime \prime}=1,2, \ldots, p
\end{array}\right)
$$

so erkennt man noch, dass die Zahlen $h_{\mu \mu^{\prime}}^{(\rho)}, h_{\mu \mu^{\prime}}^{(\sigma)}$ ohne Beschränkung der Allgemeinheit in der Form:

$$
h_{\mu \mu^{\prime}}^{(\varrho)}=p^{(\varrho)} f_{\mu}^{(\varrho)} f_{\mu^{\prime}}^{(\rho)}, \quad k_{\mu \mu^{\prime}}^{(\sigma)}=q^{(\sigma)} g_{\mu}^{(\sigma)} g_{\mu^{\prime}}^{(\sigma)} \quad\left(\begin{array}{l}
\varrho, \sigma=1,2, \ldots, n \\
\mu, \mu^{\prime}=1,2 \ldots, p
\end{array}\right)
$$

angenommen werden können, wo die $f, g$ von Null verschiedene, die $p, q$ positive garze Zahlen sind, sodass also schliesslich:

$$
a_{\mu \mu^{\prime}}^{(\varrho)}=p^{(\varrho)} f_{\mu}^{(\varrho)} f_{\mu}^{(\varrho)} a_{\mu \mu^{\prime}} \quad\left(\begin{array}{c}
\varrho=1,2, \ldots, n \\
\mu, \mu^{\prime}=1,2, \ldots, p
\end{array}\right)
$$

und :

$$
b_{\mu \mu^{\prime}}^{(\sigma)}=g^{(\sigma)} g_{\mu}^{(\sigma)} g_{\mu^{\prime}}^{(\sigma)} a_{\mu \mu^{\prime}}
$$

$$
\left(\begin{array}{r}
\sigma=1,2, \ldots, n \\
\mu, \mu^{\prime}=1,2, \ldots, p
\end{array}\right)
$$

wird. Die Gleichungen (20) stellen die gesuchten Beziehungen zwischen den Modulen des gegebenen Thetaproductes dar; sie zeigen, dass die Modulen $a_{\mu \mu^{\prime}}^{(\rho)}\left(\begin{array}{c}\rho=1,2, \ldots, n \\ \mu, \mu^{\prime}=1,2, \ldots, p\end{array}\right)$ eines Thetaproductes (1), wenn dieses durch eine $D$-Substitution überhaupt soll umgeformt werden können, aus den Modulen $a_{\mu \mu^{\prime}}$ einer einzigen Thetafunction in der Form (20) abgeleitet sein müssen, und die Gleichungen (21) sagen weiter aus, dass die. gleiche Herleitung aus den Thetamodulen $a_{\mu \mu^{\prime}}$ auch für die Modulen $b_{\mu \mu^{\prime}}^{(\sigma)}$ der neuen Thetafunctionen gilt.

Sind aber die Gleichungen (20), (21) erfüllt, so ergiebt sich aus den Gleichungen (16) für die Coefficienten $r_{\mu}^{(\rho \sigma)}$ der Substitution $D$ die Eigenschaft, dass der Ausdruck

$$
\frac{f_{\mu}^{(\varrho)}}{g_{\mu}^{(\boldsymbol{\sigma})}} r_{\mu}^{(\varrho \sigma)}
$$

für jedes $\mu$ von 1 bis $p$ den nämlichen Werth besitzt, dass also:

$$
r_{\mu}^{(\rho \sigma)}=\frac{g_{\mu}^{(\sigma)}}{f_{\mu}^{(\rho)}} t(\rho \sigma) \quad\left(\begin{array}{r}
\rho, \sigma=1,2, \ldots, n \\
\mu=1,2, \ldots, p
\end{array}\right)
$$

ist, wo die $t(\varrho \sigma)$ rationale Zahlen bezeichnen, die auf Grund der Gleichungen (15) den Bedingungen:

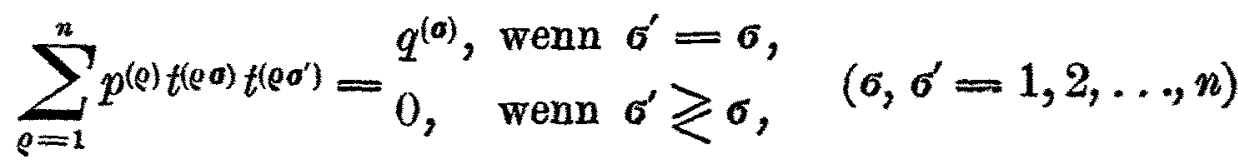

zu genügen haben. Man erhält also die allgemeinste Substitution $D$, welche ein Thetaproduct mit den Modulen (20) in ein Aggregat von Thetaproducten mit den Modulen (21) überführt, in ihren Coefficienten 
$r_{\mu}^{(\varrho \sigma)}$ durch die Gleichungen (22) gegeben, wenn man darin die $t(\rho \sigma)$ als rationale Zahlen in allgemeinster Weise so bestimmt, dass sie den Gleichungen (23) genügen, d. h. mit anderen Worten so, dass durch die Substitution:

$$
x^{(\varrho)}=\sum_{\sigma=1}^{n} t(\rho \sigma) y^{(\sigma)}
$$$$
(\varrho=1,2, \ldots, n)
$$

die Form:

in die Form:

$$
P=\sum_{\varrho=1}^{n} p^{(\varrho)} x^{(\varrho)^{2}}
$$

$$
Q==\sum_{\sigma=1}^{n} q^{(\sigma)} y^{(\sigma)^{2}}
$$

übergeht.

Beachtet man schliesslich noch, dass man die so bestimmte Substitution:

$$
m_{\mu}^{(\varrho)}=\sum_{\sigma=1}^{n} \frac{g_{\mu}^{(\sigma)}}{f_{\mu}^{(\varrho)}} t^{(\varrho \sigma)} n_{\mu}^{(\sigma)} \quad\left(\begin{array}{l}
\varrho=1,2, \ldots, n \\
\mu=1,2, \ldots, p
\end{array}\right)
$$

aus den drei Substitutionen:

$$
\begin{aligned}
& m_{\mu}^{(\varrho)}=\frac{1}{f_{\mu}^{(\rho)}} m_{\mu}^{\prime}(\rho) \\
& m_{\mu}^{\prime(\rho)}=\sum_{\sigma=1}^{n} t(\varrho \sigma) n_{\mu}^{\prime(\sigma)}, \\
& n_{\mu}^{\prime(\sigma)}=g_{\mu}^{(\sigma)} n_{\mu}^{(\sigma)}
\end{aligned}
$$$$
\left(\begin{array}{l}
\varrho=1,2, \ldots, n \\
\mu=1,2, \ldots, p
\end{array}\right)
$$$$
\left(\begin{array}{l}
\varrho=1,2, \ldots, n \\
\mu=1,2, \ldots, p
\end{array}\right)
$$$$
\left(\begin{array}{l}
\sigma=1,2, \ldots, n \\
\mu=1,2, \ldots, p
\end{array}\right)
$$

zusammensetzen kann, und dass von diesen die erste und letzte $\boldsymbol{E}$.Substitutionen specieller Art sind (sie sind auch $D$-Substitutionen, was aber hier nicht in Betracht kommt), die mittlere wieder eine $D$-Substitution ist, die aber dadurch ausgezeichnet ist, dass die Coefficientensysteme der $p$ Substitutionen (12), in welche sie zerfällt, mit einander identisch sind, so kann man sagen, dass man die allgemeine Substitution $D$ mit Hülfe der Substitutionen $E$ auf die soeben charakterisirte speciellere Form:

$$
m_{\mu}^{(\rho)}=\sum_{\sigma=1}^{n} t\left({ }^{\sigma}\right) n_{\mu}^{(\sigma)} \quad\left(\begin{array}{l}
\rho=1,2, \ldots, n \\
\mu=1,2, \ldots, p
\end{array}\right)
$$

reduciren kann. In dieser Substitution bezeichnen die $t(\rho \sigma)$ rationale Zahlen, welche den Gleichungen (22) genügen, und es wird durch sie ein Thetaproduct mit den Modulen:

$$
a_{\mu \mu^{\prime}}^{(\varrho)}=p^{(\varrho)} a_{\mu \mu^{\prime}} \quad\left(\begin{array}{r}
\varrho=1,2, \ldots, n \\
\mu, \mu^{\prime}=1,2, \ldots, p
\end{array}\right)
$$


in ein Aggregat von Thetaproducten mit den Modulen:

$$
b_{\mu \mu^{\prime}}^{(\sigma)}=q^{(\sigma)} a_{\mu \mu^{\prime}} \quad\left(\begin{array}{c}
\sigma=1,2, \ldots, n \\
\mu, \mu^{\prime}=1,2, \ldots, p
\end{array}\right)
$$

übergeführt. Bei der Aufstellung der zu einer $D$-Substitution gehörigen Thetaformel wird man sich also, nachdem die einer $E$-Substitution entsprechende Formel aus dem vorigen Artikel zu entnehmen ist, auf die specielle Form (30) dieser Substitution beschränken. Indem man aber zu dem Ende die $n^{2}$ rationalen Zahlen $t(\rho \sigma)$ auf gemeinsamen Nenner bringt, entsteht die Aufgabe, die $n p$-fach unendliche Reihe:

$$
\begin{gathered}
\vartheta\left(\left(u^{(1)}\right)\right)_{\alpha}(1) \ldots \vartheta\left(\left(u^{(n)}\right)\right)_{\alpha}(n) \\
=\sum_{[m]}^{\infty} \ldots{ }^{+\infty} \sum^{p=1} \sum_{\mu^{\prime}=1}^{p} a_{\mu \mu^{\prime}} \sum_{\varrho=1}^{n} p^{(\varrho)} m_{\mu}^{(\varrho)} m_{\mu^{\prime}}^{(\varrho)}+2 \sum_{\rho=1}^{n} \sum_{\mu=1}^{p} m_{\mu}^{(\varrho)} u_{\mu}^{(\varrho)}
\end{gathered}
$$

durch die Substitution:

$$
r m_{\mu}^{(\varrho)}=\sum_{\sigma=1}^{n} d^{(\varrho \sigma)} n_{\mu}^{(\sigma)} \quad\left(\begin{array}{l}
\varrho=1,2, \ldots, n \\
\mu=1,2, \ldots, p
\end{array}\right)
$$

umzuformen, bei der $r$ eine positive ganze Zahl, die $d^{(\rho \sigma)}$ ganze Zahlen bezeichnen, welche den Gleichungen:

$$
\sum_{\rho=1}^{n} p^{(\rho)} d(\rho \sigma) d\left(\rho \sigma^{\prime}\right)=\begin{array}{ll}
r^{2} q^{(\sigma)}, & \text { wenn } \sigma^{\prime}=\sigma, \\
0, & \text { wenn } \sigma^{\prime} \geqslant \sigma,
\end{array} \quad\left(\sigma, \sigma^{\prime}=1,2, \ldots, n\right)
$$

genügen. Diese Umformung erhält man vermittelst der am Ende des vorigen Abschnitts angeschriebenen Hauptformel $(H)$ ohne Mühe in der Gestalt:

$$
\left(r^{n} \nabla^{n-1} s\right)^{p} \vartheta\left(\left(u^{(1)}\right)\right)_{a}(1) \ldots \vartheta\left(\left(u^{(n)}\right)\right)_{a}(n)
$$

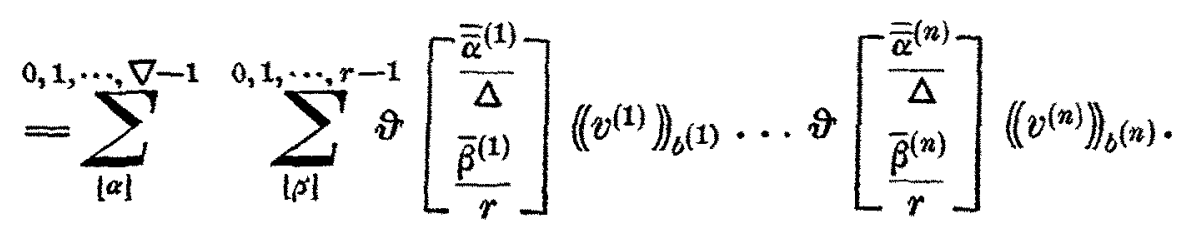

Dabei ist:

$$
a_{\mu \mu^{\prime}}^{(\varrho)}=p^{(\varrho)} a_{\mu \mu^{\prime}}, \quad b_{\mu \mu^{\prime}}^{(\sigma)}=q^{(\sigma)} a_{\mu \mu^{\prime}} ; \quad\left(\begin{array}{l}
\varrho, \sigma=1,2, \ldots, n \\
\mu, \mu^{\prime}=1,2, \ldots, p
\end{array}\right)
$$

es ist ferner:

$$
r v_{\mu}^{(\sigma)}=\sum_{\rho=1}^{n} d^{(\rho \sigma)} u_{\mu}^{(\rho)} ; \quad\left(\begin{array}{l}
\sigma=1,2, \ldots, n \\
\mu=1,2, \ldots, p
\end{array}\right)
$$

es ist weiter zur Abkürzung gesetzt: 


$$
\overline{\bar{\alpha}}_{\mu}^{(\sigma)}=r \sum_{\varrho=1}^{n} \delta^{(\varrho \sigma)} \alpha_{\mu}^{(\varrho)}, \quad \bar{\beta}_{\mu}^{(\sigma)}=\sum_{\rho=1}^{n} d^{(\varrho \sigma)} \beta_{\mu}^{(\rho)} ; \quad\left(\begin{array}{l}
\sigma=1,2, \ldots, n \\
\mu=1,2, \ldots, p
\end{array}\right)
$$

es bezeichnet $\Delta$ den Werth, $\nabla$ den absoluten Werth der Determinante $\Sigma \pm d^{(11)} d^{(22)} \ldots d^{(n n)}$ und $\delta^{(\varrho \sigma)}$ die Adjuncte von $d^{(\rho \sigma)}$ in dieser Determinante; es deutet das Zeichen $\sum_{[\alpha]}^{0,1, \ldots, \nabla-1}$ an, dass für $\begin{array}{r}\rho=1,2, \ldots, n \text { nach } \\ \mu=1,2, \ldots, p\end{array}$ jedem $\alpha_{\mu}^{(\rho)}$ von 0 bis $\nabla-1$, das Zeichen $\sum_{[\beta]}^{0,1, \ldots, r-1}$, dass für $\begin{aligned} & \rho=1,2, \ldots, n \\ & \mu=1,2, \ldots, p\end{aligned}$ nach jedem $\beta_{\mu}^{(\rho)}$ von 0 bis $r-1$ zu summiren ist; es bezeichnet endlich $s$ die nach Formel (13) des zweiten Abschnittes zu berechnende Anzahl der Normallösungen des Congruenzensystems:

$$
\sum_{\sigma=1}^{n} d(\varrho \sigma) x^{(\sigma)} \equiv 0(\bmod , r) . \quad(\varrho=1,2, \ldots, n)
$$

Bezüglich der Zusammensetzung von $D$-Substitutionen mag, da diese Frage für die späteren Untersuchungen nicht in Betracht kommt, nur kurz das Folgende bemerkt werden. Setzt man mit einer $D$-Substitution, welche ein Thetaproduct mit Modulen $a_{\mu \mu^{\prime}}^{(\sigma)}$ in ein Aggregat von Thetaproducten mit Modulen $b_{\mu \mu^{\prime}}^{(\sigma)}$ überführt, eine zweite $D$-Substitution, welche ein Thetaproduct mit den Modulen $b_{\mu_{\mu}}^{(\sigma)}$ in ein Aggregat von Thetaproducten mit Modulen $c_{\mu \mu^{\prime}}^{(\tau)}$ überführt, zusammen, so ist das Product dieser beiden Substitutionen wieder eine $D$-Substitution, und es wird durch diese ein Thetaproduct mit den Modulen $a_{\mu \mu^{\prime}}^{(o)}$ in ein Aggregat von Thetaproducten mit den Modulen $c_{\mu \mu^{\prime}}^{(\tau)}$ übergeführt. Die zwischen den Coefficienten dieser Substitutionen bestehenden Relationen sind ohne Mühe dem Vorigen zu entnehmen.

Die zur Substitution $D$ inverse Substitution:

$$
\left.D^{-1}\right) \quad n_{\mu}^{(\sigma)}=\sum_{\varrho=1}^{n} s_{\mu}^{(\varrho \sigma)} m_{\mu}^{(\varrho)}, \quad\left(\begin{array}{l}
\sigma=1,2, \ldots, n \\
\mu=1,2, \ldots, p
\end{array}\right)
$$

bei welcher, wie oben, $s_{\mu}^{(\rho \sigma)}$ die durch den Werth der Determinante $\Sigma \pm r_{\mu}^{(11)} r_{\mu}^{(22)} \ldots r_{\mu}^{(n n)}$ getheilte Adjuncte von $r_{\mu}^{(\rho \sigma)}$ in dieser Determinante bezeichnet, ist selbst wieder eine $D$-Substitution, ihre Coefficienten $s_{\mu}^{\left(\rho^{\sigma}\right)}$ genügen den leicht aus (16) erhältlichen Gleichungen:

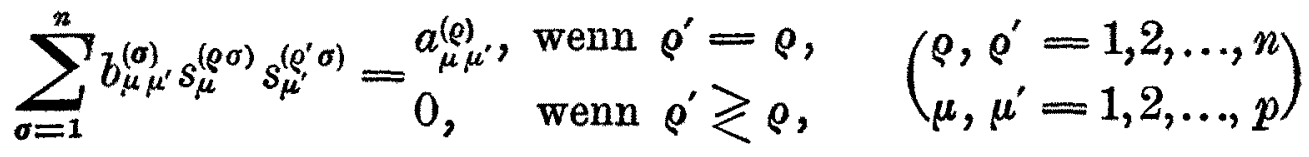

und es wird durch sie ein Thetaproduct mit den Moduln $b_{\mu^{\prime}}^{(\sigma)}$ in ein 
Aggregat von Thetaproducten mit den Modulen $a_{\mu \mu^{\prime}}^{(\rho)}$ übergeführt. Wie man die so entstehende Thetaformel aus der Formel (II) durch Umkehrung erhalten kann, ist an anderem Orte ausführlich erörtert worden*).

Endlich erkennt man obne Mühe, dass die Ueberführung eines Thetaproductes mit Modulen von der Form (20) in ein Aggregat von Thetaproducten mit Modulen von der Form (21), sobald zwischen den Thetamodulen $a_{\mu \mu^{\prime}}$ ausser den Gleichungen $a_{\mu \mu^{\prime}}=a_{\mu^{\prime} \mu}$ keine linearen Relationen bestehen, nur durch eine Substitution von der Form $D$ geschehen kann. $\mathrm{Zu}$ dem Ende beachte man, dass die Gleichungen (2) und (3), wenn man darin $a_{\mu \mu^{\prime}}^{(\varrho)}$ und $b_{\mu \mu^{\prime}}^{(\sigma)}$ durch ihre Ausdrücke aus (20), (21) ersetzt, in Folge der vorausgesetzten Unabhängigkeit der Thetamodulen $a_{\mu \mu^{\prime}}$ die Gleichungen:

$$
\begin{gathered}
\sum_{\varrho=1}^{n} p^{(\varrho)} f_{\mu}^{(\varrho)} f_{\mu^{\prime}}^{(\rho)} r_{\mu \nu}^{(\rho)} r_{\mu^{\prime} \nu^{\prime}}^{\left(\rho \sigma^{\prime}\right)}=\begin{array}{c}
q^{(\sigma)} g_{\nu}^{(\sigma)} g_{v^{\prime}}^{(\sigma)}, \text { wenn } \sigma^{\prime}=\sigma, \mu=\nu, \mu^{\prime}=\nu^{\prime} \\
0, \quad \text { in allen anderen Fällen }
\end{array} \\
\left(\begin{array}{r}
\sigma, \sigma^{\prime}=1,2, \ldots, n \\
\mu, \mu^{\prime}, v, \nu^{\prime}=1,2, \ldots, p
\end{array}\right)
\end{gathered}
$$

nach sich ziehen, und diese für $\sigma^{\prime}=\sigma, \mu^{\prime}=\mu, \nu^{\prime}=\nu$ die Gleichungen:

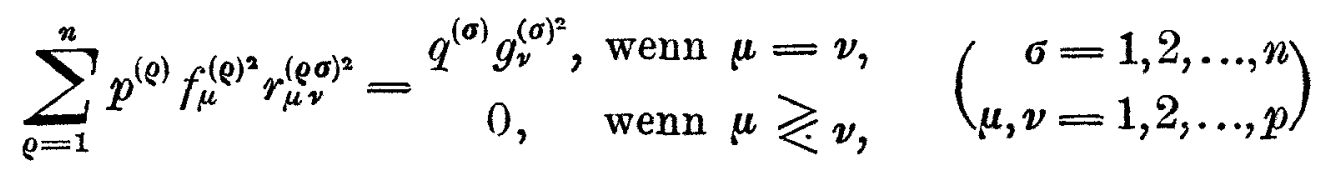

liefern. Aus diesen Gleichungen schliesst man aber, da die $p^{(\ell)}$ positive Grössen sind, sofort, dass $r_{\mu \nu}^{(\rho \sigma)}=0$ ist, sobald $\mu \gtrless \nu$ ist, womit die Substitution als eine $D$-Substitution charakterisirt ist.

4.

Zusammensetzung der allgemeinen Substitution $S$ aus Substitutionen $D$ und $E$.

Es soll jetzt nachgewiesen werden, dass die in Art. 1 aufgestellte allgemeine Substitution $S$, durch welche ein Thetaproduct mit Modulen $a_{\mu \mu^{\prime}}^{(\rho)}\left(\begin{array}{c}\varrho=1,2, \ldots, n \\ \mu, \mu^{\prime}=1,2, \ldots, p\end{array}\right)$ in ein Aggregat von Thetaproducten mit Modulen $b_{\mu \mu^{\prime}}^{(\sigma)}\left(\begin{array}{c}\sigma=1,2, \ldots, n \\ \mu, \mu^{\prime}=1,2, \ldots, p\end{array}\right)$ übergeführt wird, deren $(n p)^{2}$ Coefficienten $r_{\mu \nu}^{(\rho \sigma)}\left(\begin{array}{l}\varrho, \sigma=1,2, \ldots, n \\ \mu, \nu=1,2, \ldots, p\end{array}\right)$ also den Gleichungen:

*) Krazer und Prym, Neue Grundlagen etc. p. 22 u. f. 


$$
\begin{gathered}
\sum_{\rho=1}^{n} \sum_{\mu=1}^{p} \sum_{\mu^{\prime}=1}^{p} a_{\mu \mu^{\prime}}^{(\rho)} \gamma_{\mu \nu}^{(\rho)} r_{\mu^{\prime} \nu^{\prime}}^{\left(\rho \sigma^{\prime}\right)}=\begin{array}{c}
b_{\nu v^{\prime}}^{(\sigma)}, \text { wenn } \sigma^{\prime}=\sigma \\
0, \text { wenn } \sigma^{\prime} \geqslant \sigma
\end{array} \\
\left(\begin{array}{l}
\sigma, \sigma^{\prime}=1,2, \ldots, n \\
\nu, \nu^{\prime}=1,2, \ldots, p
\end{array}\right)
\end{gathered}
$$

genügen, immer aus Substitutionen $E$ und $D$ zusammengesetzt werden kann, in der Form:

$$
S=E_{1} D E_{2}
$$

Dabei wird nur bezüglich der Modulen $a_{\mu \mu^{\prime}}^{(\varrho)}\left(\begin{array}{c}\rho=1,2, \ldots, n \\ \mu, \mu^{\prime}=1,2, \ldots, p\end{array}\right)$ der gegebenen $n$ Thetafunctionen vorausgesetzt, dass zwischen den Modulen $a_{\mu \mu^{\prime}}^{(i)}\left(\mu, \mu^{\prime}=1,2, \ldots, p\right)$ einer einzelnen, etwa der $i^{\text {ten }}$ Thetafunction, ausser den Beziehungen $a_{\mu \mu^{\prime}}^{(i)}=a_{\mu^{\prime} \mu}^{(i)}\left(\mu, \mu^{\prime}=1,2, \ldots, p ; \mu<\mu^{\prime}\right)$ keinerlei lineare Gleichungen von der Form $\sum_{\mu=1}^{p} \sum_{\mu^{\prime}=1}^{p} k_{\mu \mu^{\prime}} a_{\mu \mu^{\prime}}^{(i)}=0$ bestehen, bei denen die $k_{\mu \mu^{\prime}}$ ganze Zahlen bezeichnen; diese Voraussetzung, welche sich zum Beweise des ausgesprochenen Satzes nothwendig erweist, steht durchaus im Einklange mit der in den vorliegenden Untersuchungen herrschenden Tendenz, Formeln aufzustellen, deren Gültigkeit nicht an besondere Specialisirungen der Thetamodulen geknüpft ist.

Wie bei anderweitigen ähnlichen Untersuchungen wird man den zu erbringenden Nachweis, dass sich jede Substitution $S$ aus Substitutionen $D$ und $E$ in der Form (42) zusammensetzen lässt, führen, indem man zeigt, dass sich jede Substitution $S$ mit Hülfe von Substitutionen $\boldsymbol{E}$ auf eine Substitution $D$ reduciren lässt in der Gestalt:

$$
E_{1}^{-1} S E_{2}^{-1}=D \text {. }
$$

Im Folgenden werden aber die Schlüsse noch etwas vereinfacht, wenn man entsprechend der mit (42) und (43) äquivalenten Gleichung:

$$
E_{2} S^{-1} E_{1}=D^{-1}
$$

und unter Beachtung des gegen Ende des vorigen Artikels bemerkten Satzes, dass die inverse einer $D$-Substitution selbst wieder eine $D$-Substitution ist, die Möglichkeit der ebengenannten Reduction an der zu $S$ inversen Substitution:

$$
n_{\nu}^{(\sigma)}=\sum_{\varrho=1}^{n} \sum_{\mu=1}^{p} s_{\mu \nu}^{(\rho \sigma)} m_{\mu}^{(\rho)}
$$$$
\left(\begin{array}{l}
\sigma=1,2, \ldots, n \\
v=1,2, \ldots, p
\end{array}\right)
$$

'nachweist, bei welcher $s_{\mu \nu}^{(\rho \sigma)}$ die durch den Werth der Determinante $\operatorname{der}(n p)^{2}$ Coefficienten der Substitution $S$ getheilte Adjuncte von $r_{\mu \nu}^{(\rho \sigma)}$ in dieser Determinante bezeichnet. Bevor nun zu diesem Nachweise selbst geschritten werden kann, müssen zwei Eigenschaften der Sub- 
stitution $S^{-1}$ aufgedeckt werden, die bei diesem Beweise eine entscheidende Rolle spielen.

Um dieselben zu erhalten, leite man zunächst aus den Gleichungen (41) ein System einfacherer Relationen ab. Zu dem Ende multiplicire man, indem man unter $\tau$ irgend eine $\mathrm{Zahl}$ aus der Reihe $1,2, \ldots, n$ und unter $\lambda$ irgend eine Zahl aus der Reihe $1,2, \ldots, p$ versteht, linke und rechte Seite der Gleichung (41) mit $s_{i y^{\prime}}^{\left(x \sigma^{\prime}\right)}$ und summire hierauf nach $\sigma^{\prime}$ von 1 bis $n$ und nach $y^{\prime}$ von 1 bis $p$. Man erhält dann, weil die Summe:

$$
\sum_{\sigma^{\prime}=1}^{n} \sum_{v=1}^{\infty} r_{u^{\prime} v^{\prime}}^{\left(\rho \sigma^{\prime}\right)} s_{2 v^{\prime}}^{\left(\tau \sigma^{\prime}\right)}
$$

nur dann einen von Null verschiedenen Werth und zwar den Werth 1 hat, wenn gleichzeitig $\mu^{\prime}=\lambda$ und $\rho=\tau$ ist, die Gleichungen:

$$
\sum_{\mu=1}^{p} a_{\mu \lambda}^{(\tau)} r_{\mu \nu}^{(\tau \sigma)}=\sum_{\nu=1}^{p} b_{\nu v^{\prime}}^{(\sigma)} s_{\lambda \nu^{\prime}}^{(\tau \sigma)}, \quad\left(\begin{array}{l}
\sigma, \tau=1,2, \ldots, n \\
\lambda, \nu=1,2, \ldots, p
\end{array}\right)
$$

aus denen jetzt weitere Schlüsse gezogen werden sollen.

Man nehme an, dass irgend eine der $x^{2}$ Determinanten:

$$
\sum \pm s_{11}^{(\rho \sigma)} s_{22}^{(\rho \sigma)} \ldots s_{p p}^{(\rho \sigma)}, \quad(0, \sigma=1,2, \ldots, n)
$$

etwa die Determinante:

$$
\sum \pm s_{11}^{(i j)} s_{22}^{(i j)} \ldots s_{p p}^{(i j)}
$$

den Werth Null besitze. Es existiren dann $p$ ganze Zahlen $k_{2}(\lambda=1,2, \ldots, p)$, die nicht alle Null sind, derart, dass gleichzeitig die $p$ Gleichungen:

$$
\sum_{\lambda=1}^{p} k_{\lambda} s_{\lambda \nu^{\prime}}^{(i j)}=0 \quad\left(\nu^{\prime}=1,2, \ldots, p\right)
$$

bestehen, und man erbält, wenn man in (45) $x=i$ und $\sigma=j$ setzt, hierauf linke und rechte Seite mit $k_{\lambda}$ multiplicirt und nach $\lambda$ von 1 bis $p$ summirt, die Gleichungen:

$$
\sum_{\lambda=1}^{p} \sum_{\mu=1}^{p} k_{\lambda} a_{\mu \lambda}^{(i)} r_{\mu \nu}^{(i i)}=0 \text {. }
$$

Aus diesen Gleichungen folgt aber, da der Voraussetzung nach zwischen den Modulen $a_{\mu \lambda}^{(i)}(\mu, \lambda=1,2, \ldots, p)$ keine linearen Relationen ausser $a_{\mu \lambda}^{(i)}=a_{2 \mu}^{(i)}$ bestehen sollen, dass die sämmtlichen Grössen: 
und ebenso die sämmtlichen Grössen:

$$
k_{\lambda} r_{\mu \nu}^{(i j)}+k_{\mu} r_{\lambda \nu}^{(i j)}=0
$$$$
(\lambda, \mu, \nu=1,2, \ldots, p)
$$

sein müssen, welche Gleichungen sofort, da nicht alle $k_{\lambda}$ Null sind, das Verschwinden der sämmtlichen $p^{2}$ Grössen $r_{\mu \nu}^{(i j)}(\mu, \nu=1,2, \ldots, p)$ nach sich ziehen. Sind aber die $p^{2}$ Grössen $r_{\mu \nu}^{(i j)}(\mu, \nu=1,2, \ldots, p)$ sämmtlich Null, so folgt aus (45), nachdem man darin $\tau=i$ und $\sigma=j$ gesetzt hat:

$$
\sum_{v^{\prime}=1}^{p} b_{\nu v^{\prime}}^{(j)} s_{\lambda v^{\prime}}^{(i j)}=0
$$$$
(\lambda, v=1,2, \ldots, p)
$$

Da nun die Determinante $\Sigma \pm b_{11}^{(j)} b_{22}^{(j)} \ldots b_{p p}^{(j)}$ als Determinante der Modulen einer Thetafunction einen von Null verschiedenen Werth besitzt, so folgt aus jenen $p$ Glejchungen, welche aus (50) hervorgehen, wenn man für $\lambda$ eine bestimmte der Zahlen $1,2, \ldots, p$ setzt und sodann für $v$ der Reihe nach die Zahlen $1,2, \ldots, p$ treten lässt, das Verschwinden der $p$ Grössen $s_{\lambda \nu^{\prime}}^{(i j)}\left(\nu^{\prime}=1,2, \ldots, p\right)$, und da dieser Schluss für $\lambda=1,2, \ldots, p$ gemacht werden kann, so ergiebt sich schliesslich als Folge der Gleichungen (50) das Verschwinden aller $p^{2}$ Grössen $s_{\mu \nu}^{(i j)}(\mu, \nu=1,2, \ldots, p)$. Man hat also endlich als erste Eigenschaft der Substitution $S^{-1}$ : Besitzt irgend eine Determinante $\Sigma \pm s_{11}^{(i j)} s_{22}^{(i j)} \ldots s_{p p}^{(i j)}$ den Werth Null, so verschwinden alle ihre $p^{2}$ Elemente $s_{\mu \nu}^{(i j)}(\mu, \nu=1,2, \ldots, p)$. Giebt man daher der Thatsache, dass die $p^{2}$ Grössen $s_{\mu \nu}^{(i j)}(\mu, \nu=1,2, \ldots, p)$ sämmtlich den Werth Null haben, dadurch Ausdruck, dass man sagt, das System der $p^{2}$ Grössen $s_{\mu \nu}^{(i j)}$ oder kürzer das System $s^{(i j)}$ sei Null, so lässt sich das soeben gefundene Resultat auch dahin umkehren, dass ein System $s^{(i j)}$, das nicht Null ist, stets eine von Null verschiedene Determinante besitzt.

Ein System $s^{(i j)}$, bei welchem alle Elemente ausserhalb der Hauptdiagonale den Werth Null haben, die Elemente der Hauptdiagonale aber den gleichen von Null verschiedenen Werth besitzen, soll fernerhin ein ,reducirtes" System heissen, und $s^{(i j)}$ in diesem Falle zugleich den gemeinsamen Werth der Elemente der Hauptdiagonale bezeichnen. Ist dann ein System $s^{(i j)}$ ein reducirtes, so folgt aus (45), nachdem man darin $\tau=i$ und $\sigma=j$ gesetzt hat:

$$
\sum_{\mu=1}^{p} a_{\mu \lambda}^{(i)} r_{\mu \nu}^{(i j)}=b_{\nu \lambda}^{(j)} s^{(i j)} . \quad(\lambda, \nu=1,2, \ldots, p)
$$

Hieraus ergiebt sich aber durch Vertauschung von $\lambda$ and $\nu$ :

$$
\sum_{\mu=1}^{p} a_{\mu \nu}^{(i)} r_{\mu \lambda}^{(i j)}=b_{\lambda \nu}^{(j)} s^{(i j)} \quad(\lambda, \nu=1,2, \ldots, p)
$$


und aus (51) und (52) wegen $b_{\lambda \nu}^{(j)}=b_{\nu \lambda}^{(j)}(\lambda, \nu=1,2, \ldots, p)$ :

$$
\sum_{\mu=1}^{p} a_{\mu \lambda}^{(i)} r_{\mu \nu}^{(i j)}=\sum_{\mu=1}^{p} a_{\mu \nu}^{(i)} r_{\mu \lambda}^{(i j)} . \quad(\lambda, \nu=1,2, \ldots, p)
$$

Da nun der gemachten Voraussetzung zu Folge zwischen den Modulen $a_{\mu \nu}^{(i)}(\mu, \nu=1,2, \ldots, p)$ ausser den Gleichungen $a_{\mu \nu}^{(i)}=a_{\nu \mu}^{(i)}$ keine linearen Relationen bestehen sollen, so folgt aus den Gleichungen (53) sofort, dass:

$$
r_{\nu \nu}^{(i j)}=r_{\lambda \lambda}^{(i j)}, \quad r_{\lambda \nu}^{(i j)}=0 \quad(\lambda, \nu=1,2, \ldots, p ; \lambda \leqslant \nu)
$$

ist, d. h. dass auch das System $\boldsymbol{r}^{(i j)}$ ein reducirtes ist, und sodann endlich aus (51):

$$
r^{(i j)} a_{\nu \lambda}^{(i)}=s^{(i j)} b_{\nu \lambda}^{(j)} .
$$$$
(\lambda, \nu=1,2, \ldots, p)
$$

Man hat daher als zweite Eigenschaft der Substitution $S^{-1}$ : Ist irgend ein System s $^{(i j)}$ ein reducirtes, d. h. ist

$$
s_{\mu \nu}^{(i j)}=\begin{gathered}
s^{(i j)}, \text { wenn } \mu=\nu, \quad(\mu, \nu=1,2, \ldots, p) \\
0, \text { wenn } \mu \geqslant \nu, \quad)
\end{gathered}
$$

wo $s^{(i j)}$ von Null verschieden ist, so sind die Modulen $a_{\mu \nu}^{(i)}$ den Modulen $b_{\mu \nu}^{(j)}$ proportional, in der Art, dass:

$$
b_{\mu \nu}^{(j)}=f^{(i j)} a_{\mu \nu}^{(i)}
$$$$
(\mu, \nu=1,2, \ldots, p)
$$

ist. Umgekehrt folgt, wenn die Gleichungen (57) erfüllt sind, aus den Gleichungen (45), nachdem man darin $\tau=i$ und $\sigma=j$ gesetzt hat:

$$
\sum_{\mu=1}^{p} a_{\mu \lambda}^{(i)} r_{\mu \nu}^{(i j)}=\sum_{\nu^{\prime}=1}^{p} f^{(i j)} a_{\nu \nu^{\prime}}^{(i)} s_{\lambda \nu}^{(i j)}, \quad(\lambda, \nu=1,2, ., ., p)
$$

und diese Gleichungen ziehen, weil zwisehen den Modulen $a_{\mu \nu}^{(i)}$ $(\mu, v=1,2, \ldots, p)$ ausser $a_{\mu \nu}^{(i)}=a_{\nu \mu}^{(i)}$ keine linearen Relationen bestehen sollen, sofort für die Systeme $r^{(i j)}$ und $s^{(i j)}$ nach sich, dass sie reducirt oder Null sind. Sind also die Modulen $a_{\mu \nu}^{(i)}$ den Modulen $b_{\mu \nu}^{(j)}$ proportional, so ist das System $s^{(i j)}$ entweder reducirt oder Null.

Um nun die verlangte, durch die Gleichung (44) dargestellte Reduction der Substitution $S^{-1}$ auszuführen, denke man sich deren Coefficienten $s_{\mu \nu}^{(\varrho \sigma)}\left(\begin{array}{l}\varrho, \sigma=1,2, \ldots, n \\ \mu, \nu=1,2, \ldots, p\end{array}\right)$ in ein quadratisches Schema angeordnet; dabei ist es aber für die folgende Untersuchung durchaus ausreichend und zweckmässig, immer ein System von $p^{2}$ Zahlen $s_{\mu \nu}^{(i j)}(\mu, \nu=1,2, \ldots, p)$ durch ein einziges Zeichen $s^{(i j)}$ zu ersetzen und dementsprechend als Repräsentanten der Coefficienten der Substitution $S^{-1}$ das Schema: 


$$
\begin{aligned}
& s^{(11)} s^{(21)} \cdots s^{(n 1)} \\
& S^{-1}=\begin{array}{l}
s^{(12)} s^{(22)} \cdots s^{(n 2)} \\
\cdot \cdot \cdot \cdot \cdot \cdot \cdot \cdot \cdot \cdot \cdot s^{(n n)}
\end{array}
\end{aligned}
$$

von nur $n$ Verticalreihen (V. R.) und $n$ Horizontalreihen (H. R.) einzuführen. Nun ordne man, indem man beachtet, dass eine Umstellung der $\nabla . R$. beziehlich der H. R. des angeschriebenen Schemas nur einer Vertauschung der Factoren in dem ursprünglichen beziehlich in den neuen Thetaproducten entspricht, die $n \mathrm{~V}$. R. und die $n \mathrm{H}$. R. von $S^{-1}$ folgendermassen.

Man suche unter den $n$ V.R. von $S^{-1}$ diejenige auf, in der am wenigsten Systeme $s^{(\rho \sigma)}$ Null sind (giebt es dabei mehrere gleichberechtigte unter den $n$ V.R., so nehme man, wie in solchen Fällen im Folgenden stets, eine beliebige darunter) und bringe sie an die erste Stelle, zugleich auch durch passende Vertauschung der H. R. die in ihr vorkommenden von Null verschiedenen Systeme, deren Anzahl $n_{1}$ sei, an die $n_{1}$ ersten Stellen, sodass also nach geschehener Anordnung die $n_{1}$ Systeme $s^{(11)}, s^{(12)}, \ldots, s^{\left(1, n_{1}\right)}$ von Null verschieden, die $n-n_{1}$ Systeme $s^{\left(1, n_{1}+1\right)}, s^{\left(1, n_{1}+2\right)}, \ldots, s^{(1, n)}$ aber Null sind. Nun betrachte man die $n_{1}$ ersten H. R., suche unter ihnen diejenige auf, in der am wenigsten Systeme $s^{(\rho \sigma)}$ Null sind, setze sie durch Umstellung der H. R. an die erste Stelle und gleichzeitig durch passende Umstellung der V. R. (mit Ausnahme der ersten, die an ihrer Stelle bleibt) die in ihr vorkommenden von Null verschiedenen Systeme an die ersten Stellen. Beträgt deren Anzahl $n_{1}^{\prime}$, so sind nach geschehener Anordnung die Systeme $s^{(11)}, s^{(21)}, \ldots, s^{\left(n_{1}^{\prime}, 1\right)}$ von Null verschieden, die Systeme $s^{\left(n_{1}^{\prime}+1,1\right)}, s^{\left(n_{1}^{\prime}+2,1\right)}, \ldots, s^{(n, 1)}$ aber Null.

Nun bezeichne man für $\sigma=1,2, \ldots, n_{1}$ mit $\sigma_{\mu v}^{(1 \sigma)}$ die durch den Werth der Determinante des Systems $s^{(1 \sigma)}$ dividirte Adjuncte von $s_{\mu \nu}^{(1 \sigma)}$ in dieser Determinante und bilde eine $E$-Substitution:

$$
x_{\mu}^{(\varrho)}=\sum_{\nu=1}^{p} r_{\mu \nu}^{(\varrho)} y_{\nu}^{(\varrho)}
$$$$
\left(\begin{array}{l}
\varrho=1,2, \ldots, n \\
\mu=1,2, \ldots p
\end{array}\right)
$$

dadurch, dass man für $\varrho=1,2, \ldots, n_{1}$ :

$$
r_{\mu \nu}^{(\varrho)}=\sigma_{\mu \nu}^{(1 \rho)} \text {, }
$$

$$
(\mu, \nu=1,2, \ldots, p)
$$

für $\varrho=n_{1}+1, n_{1}+2, \ldots, n$ aber:

$$
r_{\mu \nu}^{(\varrho)}=\begin{aligned}
& 1, \text { wenn } \mu=\nu, \\
& 0, \text { wenn } \mu \geqslant \nu, \quad(\mu, \nu=1,2, \ldots, p)
\end{aligned}
$$

setzt. Die so gebildete $E$-Substitution soll $E_{2}$ heissen. Man bezeichne ebenso für $\varrho=1,2, \ldots, n_{1}^{\prime}$ mit $\sigma_{\mu \nu}^{(\rho)}$ die durch den Werth der 
Determinante des Systems $s^{\left(\varrho^{1)}\right.}$ dividirte Adjuncte von $s_{\mu \nu}^{\left(\rho^{1)}\right.}$ in dieser Determinante und bestimme eine weitere $E$-Substitution, $E_{1}$, dadurch, dass man in (59) für $\varrho=2,3, \ldots, n_{1}^{\prime}$ :

$$
r_{\mu \nu}^{(\rho)}=\sigma_{\mu \nu}^{(\rho 1)}, \quad(\mu, \nu=1,2, \ldots, p)
$$

für $\varrho=1$ und $\varrho=n_{1}^{\prime}+1, n_{1}^{\prime}+2, \ldots, n$ aber:

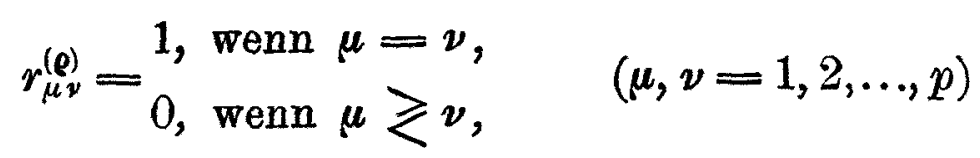

setzt. Setzt man dann diese Substitutionen $E_{2}$ und $E_{1}$ mit der Substitution $S^{-1} \mathrm{zu}$ einer Substitution $T$ zusammen in der Form:

$$
T=E_{2} S^{-1} E_{1}
$$

so ist in der Substitution $T$, wenn deren Coefficienten mit:

bezeichnet werden:

$$
\left(\begin{array}{l}
\rho, \sigma=1,2, \ldots, n \\
\mu, \nu=1,2, \ldots, p
\end{array}\right)
$$

für $\sigma=1,2, \ldots, n_{1}$ :

$$
t_{\mu \nu}^{(1 \sigma)}=\begin{aligned}
& 1, \text { wenn } \mu=\nu, \\
& 0, \text { wenn } \mu \gtrless \nu,
\end{aligned}
$$

für $\sigma=n_{1}+1, n_{1}+2, \ldots, n$ :

$$
t_{\mu \nu}^{(1 \sigma \sigma}=0
$$

für $\varrho=1,2, \ldots, n_{1}^{\prime}$ :

$$
(\mu, \nu=1,2, \ldots, p)
$$

$$
t_{\mu \nu}^{(\varrho 1)}=\begin{aligned}
& 1, \text { wenn } \mu=v, \\
& 0, \text { wenn } \mu \geq v,
\end{aligned}
$$

für $\varrho=n_{1}^{\prime}+1, n_{1}^{\prime}+2, \ldots, n$ :

$$
t_{\mu \nu}^{(01)}=0 \text {. }
$$

Nun sind aber nach Früherem, sobald ein System $t^{(1 \sigma)}$ reducirt ist, die Modulen $a_{\mu \mu^{\prime}}^{(1)}$ den Modulen $b_{\mu \mu^{\prime}}^{(\boldsymbol{a})}\left(\mu, \mu^{\prime}=1,2, \ldots, p\right)$ proportional, und ebenso, wenn ein System $t^{\left(\rho^{1)}\right.}$ reducirt ist, die Modulen $a_{\mu^{\prime}}^{(\rho)}$ den Modulen $b_{\mu \mu^{\prime}}^{(1)}$ proportional, und da bei der Substitution $T$ ersteres für $\sigma=1,2, \ldots n_{1}$, letzteres für $\varrho=1,2, \ldots, n_{1}^{\prime}$ gilt, so folgt endlich, dass bei ihr für jedes $\rho$ von 1 bis $n_{1}^{\prime}$ und jedes $\sigma$ von 1 bis $n_{1}$ die Modulen $a_{\mu \mu^{\prime}}^{(\varrho)}$ den Modulen $b_{\mu \mu^{\prime}}^{(\sigma)}$ proportional sind, was wiederum nach Früherem nach sich zieht, dass die sämmtlichen $n_{1} n_{1}^{\prime}$ Systeme $t^{(\varrho \sigma)}\left(\varrho=1,2, \ldots, n_{1}^{\prime}, \sigma=1,2, \ldots, n_{1}\right)$ reducirt oder Null sind*).

*) Es kann nicht stören, dass hier die Modulen $a_{\mu \mu^{\prime}}^{(\rho)}$ und $b_{\mu \mu^{\prime}}^{(\sigma)}$ in anderer Bedeutung eingeführt werden als früher; hier ist angenommen, dass durch die 
Ist nun $n_{1}=n_{1}^{\prime}=n$, so ist bereits die Substitution $T$ eine $D$-Substitution, da bei ihr alle Coefficienten $t_{\mu \nu}^{(\rho)}$, bei denen $\mu \gtrless \nu$ ist, verschwinden, und die Gleichung (60) stellt die verlangte Reduction der Substitution $S^{-1}$ dar. Ist dagegen $n_{1}$ oder $n_{1}^{\prime}$, oder sind beide Zahlen kleiner als $n$, so bat man die Reduction in der jetzt zu erörternden Weise fortzusetzen.

Man nehme zunächst an, es seien $n_{1}$ and $n_{2}$ beide kleiner als $n$. Die erste $\nabla . R$. von $T$ hat an den $n-n_{1}$ letaten Stellen lauter Systeme, die Null sind; man suche unter der $2^{\text {ten }}, 3^{\text {ten }}, \ldots, n_{1}{ }^{\text {ten }} V . R$. jene auf, bei welcher von den $n-n_{1}$ letzten Systemen die wenigsten Null sind, und bringe dieselbe durch Umstellung der $\nabla$. R. an die $n_{1}^{\prime}$ te Stelle, gleichzeitig aber durch passende Umstellung der $n-n_{1}$ letzten $H$. $\mathrm{R}$. diese von Null verschiedenen Systeme, deren Anzahl $n_{2}-n_{1}$ betrage, an die $n_{1}+1^{\text {te }}, n_{1}+2^{\text {te }}, \ldots, n_{2}$ te Stelle. Hierauf suche man unter der $n_{1}+1^{\text {ten }}, n_{1}+2^{\text {tee }}, \ldots n_{2}^{\text {ten }}$ H. R. diejenige auf, bei der unter den $n-n_{1}^{\prime}$ letzten Systemen die wenigsten Null sind, und bringe sie durch Umstellung der H. R. an die $n_{1}+1^{\text {te }}$ Stelle, gleichzeitig aber durch passende Umstellung der $n-n_{1}^{\prime}$ letzten $V$. $R$. diese von Null verschiedenen Systeme, deren Anzahl $n_{2}^{\prime}-n_{1}^{\prime}$ betrage, an die $n_{1}^{\prime}+1^{\text {te }}, n_{1}^{\prime}+2^{\text {te }}, \ldots, n_{2}^{\prime}$ te Stelle. Es sind dann nach geschehener Anordnung bei der Substitution $T$ in der $n_{1}{ }^{\prime}$ ten $\nabla$. R. die Systeme $t^{\left(n_{1}^{\prime}, n_{1}+1\right)}, t^{\left(n_{1}^{\prime}, n_{1}+2\right)}, \ldots, t^{\left(n_{1}^{\prime}, n_{2}\right)}$ ron Null verschieden, die Systeme $t^{\left(n_{1}^{\prime}, n_{2}+1\right)}, t^{\left(n_{2}^{\prime}, n_{2}+2\right) !}, \ldots, t^{\left(n^{\prime}, n\right)}$ Null, und in der $n_{1}+1^{\text {ten }}$ H. R, die Systeme $t^{\left(n_{2}{ }^{\prime}, n_{2}+1\right)}, t^{\left(n_{1}{ }^{\prime}+1, n_{1}+1\right)}, \ldots, t^{\left(n_{2}{ }^{\prime}, n_{1}+1\right)}$ von $N$ all verschieden, die Systeme $t^{\left(n_{2}^{\prime}+1, n_{1}+1\right)}, t^{\left(n_{2}^{\prime}+2, n_{1}^{\prime}+1\right)}, \ldots, t^{\left(n, n_{2}+1\right)}$ Null. Nun bestimme

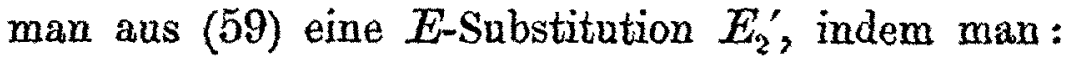

für $\varrho=1,2, \ldots, n_{1}$ :

$$
r_{\mu \nu}^{(\rho)}=\begin{aligned}
& 1, \text { wenn } \mu=v, \\
& 0, \text { wenn } \mu \geqslant \nu,
\end{aligned}
$$

für $\rho=n_{1}+1, n_{1}+2, \ldots, n_{2}$ :

$$
r_{\mu \nu}^{(\rho)}=\tau_{\mu \nu}^{\left(n_{2}^{\prime}, \rho\right)},
$$

für $\rho=n_{2}+1, n_{2}+2, \ldots, n$ :

$$
r_{\mu \nu}^{(\rho)}=\begin{aligned}
& 1, \text { wenn } \mu=\nu, \\
& 0, \text { wenn } \mu \gtrless \nu,
\end{aligned}
$$

setzt, unter $\tau_{\mu \nu}^{\left(n_{1}, \rho\right)}$ die durch die Determinante des Systems $t^{\left(n_{1}^{\prime}, \varphi\right)}$ getheilte Adjuncte von $t_{\mu \nu}^{\left(n_{i}, \rho\right)}$ in dieser Determinante verstanden, and eine weitere $E$-Substitution $E_{1}^{\prime}$, indem man in (59):

Substitution $T$ ein Thetaproduct mit den Modulen $a_{\mu \mu^{\prime}}^{(\varrho)}$ in ein Aggregat von Thetaproducten mit den Modulen $b_{\mu \mu^{\prime}}^{(a)}$ übergeführt werde. 
für $\varrho=1,2, \ldots, n_{1}^{\prime}$ :

$$
r_{\mu \nu}^{(\rho)}=\begin{aligned}
& 1, \text { wenn } \mu=\nu, \\
& 0, \text { wenn } \mu \gtrless \nu,
\end{aligned}
$$

für $\varrho=n_{1}^{\prime}+1, n_{1}^{\prime}+2, \ldots, n_{2}^{\prime}$ :

für $\varrho=n_{2}^{\prime}+1, n_{2}^{\prime}+2, \ldots, n$ :

$$
r_{i v y}^{(\ell)}=\tau_{\mu \nu}^{\left(0, n_{i}+1\right)}
$$

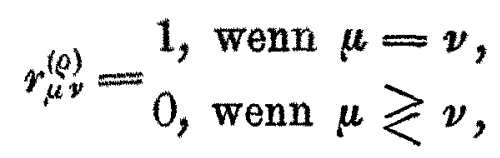

setzt, unter $\tau_{\mu \nu}^{\left(0, n_{1}+1\right)}$ die durch die Determinante des Systems $t^{\left(0, n_{1}+1\right)}$ getheilte Adjuncte von $t_{\mu \nu}^{\left(\rho, n_{1}+1\right)}$ in dieser Determinante verstanden. Setat man dann diese Substitutionen $E_{2}^{\prime}, E_{1}^{\prime}$ mit der Substitution $T$ zu einer Substitution $V$ zusammen in der Form:

$$
U=E_{2}^{\prime} T E_{1}^{\prime} \text {, }
$$

so sind in der Substitution $U$ die Systeme $u^{\left(n_{1}^{\prime}, n_{1}+1\right)}, u^{\left(n_{1}{ }^{\prime}, n_{1}+2\right)}, \ldots, u^{\left(n_{1}^{\prime}, n_{2}\right)}$ und ebenso die Systeme $u^{\left(n_{1}^{\prime}+1, n_{2}+1\right)}, u^{\left(n_{1}^{\prime}+2, n_{1}+1\right)}, \ldots, u^{\left(n_{2}^{\prime}, n_{1}+1\right)}$ reducirt, und man schliesst daraus nicht nur, wie früher, dass für jedes $\varrho$ von $n_{1}^{\prime}$ bis $n_{2}^{\prime}$ und für jedes $\sigma$ von $n_{1}+1$ bis $n_{2}$ die Modulen $a_{\mu \mu^{\prime}}^{(\rho)}$ den Modulen $b_{\mu \mu^{\prime}}^{(\sigma)}$ proportional and daher alle Systeme $u^{(\ell \sigma)}$, welche diesen Werthen von $\varrho$ and $\sigma$ entsprechen, reducirt oder Null sind, sondern in Verbindung mit dem frither erhaltenen Resultate sofort weiter, dass die Proportionalität der Modulen $a_{\mu \mu^{\prime}}^{(\varrho)}$ mit den Modulen $b_{\mu \mu^{\prime}}^{(\sigma)}$ für jedes $\varrho$ vou 1 bis $n_{2}^{\prime}$ und jedes $\sigma$ von 1 bis $n_{2}$ besteht, and dass daher alle $n_{2}^{\prime} n_{2}$ den ersten $n_{2}$ H. R. und ersten $n_{2}^{\prime}$ V. R. gemeínsamen Systeme $u^{(\varrho \sigma)}\left(\varrho=1,2, \ldots, n_{2}^{\prime} ; \sigma=1,2, \ldots, n_{2}\right)$ reducirt oder Nall sind.

Das angegebene Verfahren ist auch anwendbar, wenn $n_{1}^{\prime}=n$ ist, in welchem Falle sich die Substitution $E_{1}^{\prime}$ anf die identische reducirt; es versagt aber, wenn $n_{1}=n$ ist, und ferner, wenn die $n_{1}^{\prime}$ ersten V. R. von $T$ an ihren $n-n_{1}$ letzten Stellen lauter Systeme haben, die Null sind. In beiden Fällen kann, wie man leicht sieht, die Reduction von $T$ in der Weise vorgenommen werden, dass man das im Vorigen angegebene Reductionsverfahren unter durchgängiger Vertauschung der H. R. mit den V.R. nachbildet.

Ist nun für die Substitution $U n_{2}=n_{2}{ }^{\prime}=n$, so ist sie- eine $D$. Substitution, and es wird durch die aus (60) and (61) folgende Gleichung:

$$
U=E_{2}^{\prime} E_{2} S^{-1} E_{1} E_{1}^{\prime}
$$

die verlangte Reduction der Substitution $S^{-1}$ geliefert, wenn man noch die beiden $E$-Sabstitutionen $E_{2}^{\prime}$ and $E_{2}^{*}$ and ebenso die beiden $E$-Substitutionen $E_{1}$ und $E_{1}^{\prime}$ zu je einer $E$-Substitution zusammensetzt. Sind 
dagegen $n_{2}$ und $n_{2}^{\prime}$ oder eine dieser beiden Zahlen kleiner als $n$, so hat man das Reductionsverfahren in der angegebenen Weise fortzusetzen, bis man schliesslich zu einer $D$-Substitution gekommen ist.

Man hat so den im Vorigen ausgesprochenen Satz bewiesen, dass man jede beliebige Substitution $S$, durch welche ein Product von $n$ Thetafunctionen in ein Aggregat solcher Producte übergeführt wird, stets aus Substitutionen $D$ und $E$ in der Form (42) zusammensetzen kann.

Dieses Resultat lässt sich ausführlicher folgendermassen aussprechen.

Wenn ein Thetaproduct (1) mit den Modulen $a_{\mu \mu^{\prime}}^{(o)}\left(\begin{array}{r}o=1,2, \ldots, n \\ \mu, \mu^{\prime}=1,2, \ldots, p\end{array}\right)$ durch irgend eine lineare Substitution $S$ mit rationalen Coefficienten in ein Aggregat von Thetaproducten mit den Modulen $b_{v v^{\prime}}^{(o)}\left(\begin{array}{c}\sigma=1,2, \ldots, n \\ \nu, \nu^{\prime}=1,2, \ldots, p\end{array}\right)$ übergeführt werden kann, wozu die nothwendigen und hinreichenden Bedingungen in den Gleichungen (2) und (3) angegeben sind, so kann man sowohl das gegebene Thetaproduct mit den Modulen $a_{\mu, \mu^{\prime}}^{(\varrho)}$ als auch das Thetaproduct mit den Modulen $b_{v v^{\prime}}^{(\sigma)}$ durch je eine Substitution $E$, d. h. durch blosse Umformung jeder einzelnen seiner Thetafunctionen vermittelst einer linearen Substitution, in ein Aggregat von Thetaproducten überführen, deren Modulen ganze Vielfache $p^{(\varrho)} a_{\mu \mu^{\prime}}$ bez. $q^{(\sigma)} a_{v v^{\prime}}$ der Modulen einer einzigen Thetafunction sind, so dass nunmehr jedes Thetaproduct mit den Modulen $p^{(\rho)} a_{\mu \mu^{\prime}}$ durch eine Substitution von der Art $D$ in Thetaproducte mit den Modulen $q^{(\sigma)} a_{v v^{\prime}}$ übergeht.

Aus der Möglichkeit der Zusammensetzung der allgemeinen Substitution $S$ aus Substitutionen $D$ und $E$ folgt nun weiter, dass man ebenso jene Thetaformel, welche die durch die allgemeine Substitution $S$ bewirkte Ueberführung eines Thetaproductes in ein Aggregat neuer Thetaproducte zum Ausdruck bringt, aus den in diesem Abschnitte aufgestellten, den Substitutionen $E$ und $D$ entsprechenden Formeln $(I),(I I)$ durch Zusammensetzung erhalten kann, dass man sich also hinsichtlich der Aufstellung von Thetaformeln auf diese beiden Formeln beschränken darf. 


\section{Vierter Abschnitt.}

\section{Veber die zu den Substitutionen $E$ und $D$ gehörigen Theta-} formeln. Specielle Fälle derselben. Historisches.

1.

Deber die zu den Substitutionen $E$ gehörigen Thetaformeln.

Die der allgemeinen Substitution $E$ entsprechende Thetaformel $(I)$ ist zuerst von Herrn Prym und mir*) angegeben worden; bis dahin waren nur ganz specielle Fälle derselben bekannt, welche alle der Annahme entsprechen, dass bei der zu Grunde liegenden Substitution (7) sämmtliche nicht in der Hauptdiagonale stehenden Coefficienten $e_{\mu \nu}$ den Werth Null haben. Ist aber für jedes $\mu$ und $\nu$ von 1 bis $p e_{\mu \nu}=0$, sobald $\mu \gtrless \nu$, dagegen $e_{11}=e_{22}=\cdots=e_{p p}=q$, wo $q$ eine positive ganze Zahl bezeichnet, die zu $r$ relativ prim ist, so nimmt die Formel $(I)$ die einfache Gestalt $*$ ):

$$
r^{p} \mathcal{V}((u))_{a}=\sum_{\rho_{1}, \cdots, o_{p}}^{0,1, \cdots, q-1} \sum_{\sigma_{c}, \cdots, \sigma_{p}}^{0,1, \cdots, r-1} \mathcal{v}\left[\begin{array}{c}
\frac{r \varrho}{q} \\
\frac{q \sigma}{r}
\end{array}\right]((v))_{b}
$$

an, bei der die Grössen $v, b$ jetzt mit den Grössen $u, a$ durch die Gleichungen:

$$
v_{\nu}=\frac{q}{r} u_{v}, \quad b_{v v^{\prime}}=\frac{q^{2}}{r^{2}} a_{v v^{\prime}} \quad\left(v, v^{\prime}=1,2, \ldots, p\right)
$$

verknüpft sind, und aus der weiter für $r=1$ die Formel $\left.{ }^{* * *}\right)$ :

$$
v\left((u)_{\alpha}=\sum_{\rho_{1}, \cdots, \varrho_{p}}^{0,1, \cdots, q-1} v\left[\begin{array}{l}
\frac{e}{q} \\
0
\end{array}\right]\left((q u)_{q^{2} a},\right.\right.
$$

für $q=1$ dagegen die Formel $\dagger$ ):

$$
r^{p} \vartheta((u))_{\alpha}=\sum_{\sigma_{1}, \cdots, \sigma_{p}}^{0,1, \cdots, r-1} \vartheta\left[\begin{array}{c}
0 \\
\frac{\sigma}{r}
\end{array}\right]\left(\left(\frac{u}{r}\right)\right)_{\frac{a}{r^{2}}}
$$

hervorgeht Dabei mag noch bemerkt werden, dass die Formeln (3) und (4) nicht wesentlich versehieden von einander sind, insofern als jede von ihnen als die Umkehrung der anderen angesehen werden kann. Lässt man nämlich, indem man unter $\sigma_{1}, \ldots, \sigma_{p}$ ganze Zahlen

*) Krazer und Prym, Neue Grundlagen etc., pag. 72, Formel $\left(I_{0}\right)$.

**) a. a. 0 . pag. 77 , Formel $\left(I_{2}\right)$.

***) a. a. O. pag. 77, Formel $\left(\mathrm{I}_{3}\right)$.

†) a. a. 0. pag. 77 , Formel $\left(\bar{I}_{3}\right)$. 
versteht, in der Formel (3) für $\mu=1,2, \ldots, p u_{\mu}$ in $u_{\mu}+\frac{1}{q} \sigma_{\mu} \pi i$ übergehen, so entsteht unter Anwendung bekannter Hülfsformeln ${ }^{*}$ ) die allgemeinere Formel:

$$
\vartheta\left[\begin{array}{l}
0 \\
\frac{\sigma}{q}
\end{array}\right]((u))_{a}=\sum_{\rho_{1}, \cdots, \rho_{p}}^{0,1, \ldots, q-1} \vartheta\left[\begin{array}{l}
\varrho \\
q \\
0
\end{array}\right]\left((q u)_{q^{2} a} e^{\frac{2 \pi i}{q} \sum_{\mu=1}^{p} \rho_{\mu} \sigma}\right.
$$

Diese Formel enthält $q^{p}$ verschiedene specielle, die man erhält, wenn man jeder der $p$ Grössen $\sigma_{1}, \ldots, \sigma_{p}$ unabhängig von den anderen die Werthe $0,1, \ldots, q-1$ ertheilt. Addirt man diese $q^{p}$ Gleichungen zu einander, d. h. summirt man in (5) über jedes $\sigma$ von 0 bis $q-1$, so entsteht, da die Summe:

$$
\sum_{\sigma_{1}, \cdots, \sigma_{p}}^{0,1, \cdots, q-1} e^{\frac{2 \pi i}{q} \sum_{\mu=1}^{p} o_{\mu} \sigma_{\mu}}
$$

nur dann einen von Null verschiedenen Werth und zwar den Werth $q^{p}$ besitzt, wenn $\varrho_{1}=\varrho_{2}=\cdots=\varrho_{p}=0$ ist, eine Formel, die sich von der Formel (4) ersichtlich nur durch die Bezeichnung unterscheidet. In ähnlicher Weise kann man von der Formel (4) zu der Formel (3) zurückkehrea.

Die Formeln (3) und (4) sind für den Fall $p=1$ längst bekannt. Schon Jacobi*) hat bemerkt, dass die $\vartheta(u)_{a}$ darstellende Reihe, wenn man in ihr die geraden Glieder von den ungeraden trennt, in zwei Reihen zerfällt, von denen jede für sich eine Thetafunction mit dem Argumente $2 u$ und dem Modul $4 a$ darstellt, und ist so zu der Formel (3) für $p=1$ und $q=2$ gelangt. Schröter $* *$ ) hat diese Zerspaltung einer Thetareihe in mebrere durch Zusammenfassung derjenigen Glieder, bei denen die Summationsbuchstaben einander nach dem Modul $q$ congruent sind, auf den Fall eines beliebigen $q$ ausgedehnt und so die Formel (3) für $p=1$ and beliebiges $q$ erhalten, während die Formel (4) zuerst von Herrn Gordan $\dagger$ ) angegeben wurde. Die Formeln (3) und (4) sind für den Fall $p=1$ genau jene Umformungen der Thetareihe, welche am Ende des ersten Abschnittes für eine beliebige einfach unendliche Reihe angeschrieben sind.

Nachdem die Formeln (3) und (4) für den Fall $p=1$ gefunden

*) a. a. O. pag. 7, Formeln (A) und (D).

**) Jacobi, Theorie der elliptischen Functionen aus den Eigenschaften der Thetareihen abgeleitet. Ges. Werke Bd. 1, pag. 515. 1854, pag. 9.

**) Schröter, De aequationibus modularibus. Inaug. Diss. Königsberg

†) Gorda n, Beziehungen zwischen Thetaproducten. J. für Math. Bd. 66 (1866), pag. 191. 
waren, war es leicht zu ersehen, dass solche Formeln auch für Thetafunctionen mehrerer Veränderlichen bestehen; sie wurden zuerst (unter Beschränkung auf den Fall $p=2$ und $q=2$ ) von Herrn Königsberger*) angegeben, doch hatte schon vorher Herr Thomae**) die Formel :

bei der

$$
\vartheta((u))_{a}=\sum_{\varrho_{1}=0}^{q_{2}-1} \ldots \sum_{\varrho_{p}=0}^{q_{p}-1} \vartheta\left[\begin{array}{l}
\frac{\varrho}{q} \\
0
\end{array}\right]\left((v)_{b}\right.
$$

$$
v_{v}=q_{v} u_{v}, \quad b_{v v^{\prime}}=q_{v} q_{v^{\prime}} a_{v v^{\prime}} \quad\left(\nu, v^{\prime}=1,2, \ldots, p\right)
$$

ist, aufgestellt; eine Formel, welche allgemeiner als die Formel (3) ist, in die sie für $q_{1}=q_{2}=\cdots=q_{p}=q$ übergeht, und welche aus der Formel (I) erhalten wird, wenn man darin $r=1$ und:

setat.

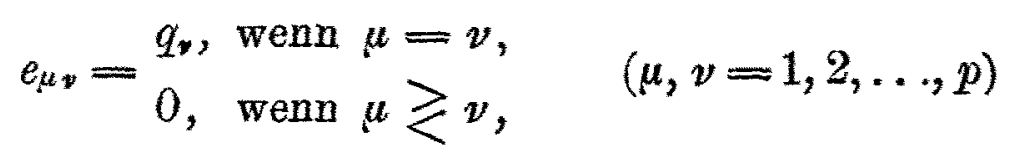

Was endlich den Charakter der Formel (I) angeht, so liegt derselben, wie Herr Prym und ich ***) gezeigt haben, eine lineare Transformation der Perioden mit gebrochenen Transformationszahlen zu Grunde, also eine solche, bei der das neue $\mathrm{Pe}$ riodengitter Periodenparallelotope desselben Inhalts, aber nicht die näm* lichen Gitterpunkte aufweist, wie das ursprüngliche. Die Formel (1) entspricht z. B. im Falle $p=1$ der

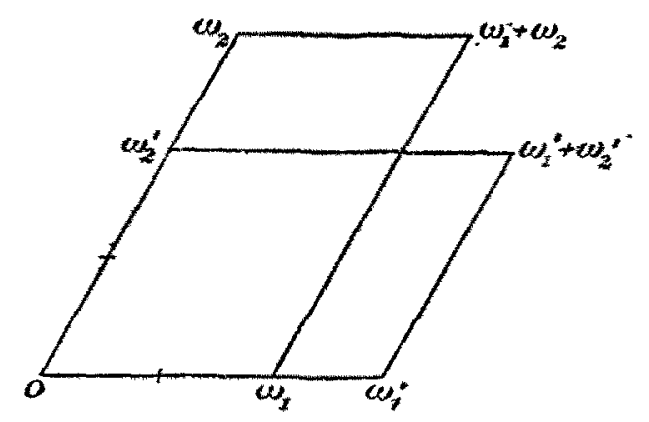
Periodentransformation:

$$
\omega_{1}=\frac{q}{r} \omega_{1}^{\prime}, \quad \omega_{2}=\frac{r}{q} \omega_{2}^{\prime},
$$

für welche unter der Annahme $q=2, r=3$ die Periodenparallelogramme durch nebenstehende Figur dargestellt werden.

2.

Ueber die zu den Substitutionen $D$ gehörigen Thetaformeln.

Die der allgemeinen Substitution $D$ entsprechende Thetaformel (II) ist gleichfalls von Herrn Prym und mir $\dagger$ ) aufgestellt worden. Die

*) Königsberger, Ueber die Transformation der Abel'schen Functionen erster Ordunng. J. für Math., Bd. 64 (1865), pag. 33.

**) Thomae, Die allgemeine Transformation der $\Theta$-Functionen mit beliebig vielen Variabeln. Inaug,-Diss. Göttingen 1864, pag. 5.

***) Krazer und Prym, Neue Grundlagen etc. pag. 73 u. f.

†) Krazer und Prym, Neue Grundlagen etc., pag. 20, Formel $(\theta)$. 
zuerst bekannt gewordenen speciellen Fälle der Formel bezogen sich ausschliesslich auf den Fall $n=2$, für welchen Schröter*) die bekannte, der Substitution:

$$
\begin{aligned}
& m_{\mu}^{(1)}=\beta n_{\mu}^{(1)}+n_{\mu}^{(2)} \\
& m_{\mu}^{(2)}=-\alpha n_{\mu}^{(1)}+n_{\mu}^{(2)}
\end{aligned}
$$

$$
(\mu=1,2, \ldots, p)
$$

entsprechende Formel:

(9) $\vartheta\left(\left(u^{(1)}\right)\right)_{\alpha}(1) \vartheta\left(\left(u^{(2)}\right)\right)_{\alpha^{(2)}}=\sum_{\varepsilon_{1}, \cdots, \varepsilon_{p}}^{0,1, \cdots, \alpha+\beta-1} \vartheta\left[\begin{array}{c}\varepsilon \\ \alpha+\beta \\ 0\end{array}\right]\left(\left(v^{(1)}\right)\right)_{b}(1) \vartheta\left[\begin{array}{c}\alpha \varepsilon \\ \alpha+\beta \\ 0\end{array}\right]\left(\left(v^{(2)}\right)\right)_{b}(2)$ bei der:

$$
\begin{array}{ll}
a_{\mu \mu^{\prime}}^{(1)}=\alpha a_{\mu \mu^{\prime}}, & b_{\mu \mu^{\prime}}^{(1)}=\alpha \beta(\alpha+\beta) a_{\mu \mu^{\prime}} \\
a_{\mu \mu^{\prime}}^{(2)}=\beta a_{\mu \mu^{\prime}}, & b_{\mu \mu^{\prime}}^{(2)}=\left(\alpha, \mu^{\prime}=1,2, \ldots, p\right)
\end{array}
$$

ist, während die Variablen $v$ mit den Variablen $u$ durch die Gleichungen:

$$
\begin{aligned}
& v_{\mu}^{(1)}=\beta u_{\mu}^{(1)}-\alpha u_{\mu}^{(2)} \\
& v_{\mu}^{(2)}=u_{\mu}^{(1)}+u_{\mu}^{(2)}
\end{aligned} \quad(\mu=1,2, \ldots, p)
$$

zusammenhängen, für $p=1$ angegeben hat**). Später hat Schrö$\operatorname{ter}^{* *}$ ) die Formel (9) durch die allgemeinere, der Substitution:

$$
\begin{aligned}
& m_{\mu}^{(1)}=\operatorname{tr} n_{\mu}^{(1)}+s n_{\mu}^{(2)} \\
& m_{\mu}^{(2)}=-s p n_{\mu}^{(1)}+t n_{\mu}^{(2)}
\end{aligned} \quad(\mu=1,2, \ldots, p)
$$

und Hoppe†) durch die noch allgemeinere, der Substitution:

$$
\begin{aligned}
& m_{\mu}^{(1)}=f h c n_{\mu}^{(1)}-g e d n_{\mu}^{(2)} \\
& m_{\mu}^{(2)}=g h a n_{\mu}^{(1)}+f e b n_{\mu}^{(2)}
\end{aligned} \quad(\mu=1,2, \ldots, p)
$$

entsprechende ersetzt.

*) Schröter, De aequationibus modularibus. Inaug.-Diss. Königsberg 1854, pag. 7, Formel (1).

**) Für $p>1$ finden sich solche Formeln zuerst bei Königsberger, Ueber die Transformation der Abel'schen Functionen erster Ordnung. J. für Math. Bd. 64 (1865), pag. 24.

***) Schröter, Deber die Entwicklung der Potenzen der elliptischen Transcendenten $\checkmark$ und die Theilung dieser Functionen. Hab.-Schrift, Breslau 1855, pag. 6, Formel 5.

†) Hoppe, Verallgemeinerang einer Relation der Jacobi'sehen Functionen. Archiv f. Math. u. Phys. Th. 70 (1884), pag. 403, Formel (9). Die von Herrn Huebner, Ueber die Umformung unendlicher Reihen und Producte mit Beziehung auf die Theorie der elliptischen Functionen (Progr. Königsberg 1891) angegebenen Formeln (I) - (VI) pag. 37 u. f. entstehen aus solchen Formeln in Verbindung mit Formeln (4). 
Geht man nun auf den Fall eines beliebigen $n$ über, so erhält man unter der Annahme $r=1$ zu der Substitution:

$$
m_{\mu}^{(\varrho)}=\sum_{\sigma=1}^{n} d^{(\varrho \sigma)} n_{\mu}^{(\sigma)}, \quad\left(\begin{array}{l}
\varrho=1,2, \ldots, n \\
\mu=1,2, \ldots, p
\end{array}\right)
$$

bei welcher die ganzen Zahlen $\partial(\rho \sigma)$ den Gleichungen:

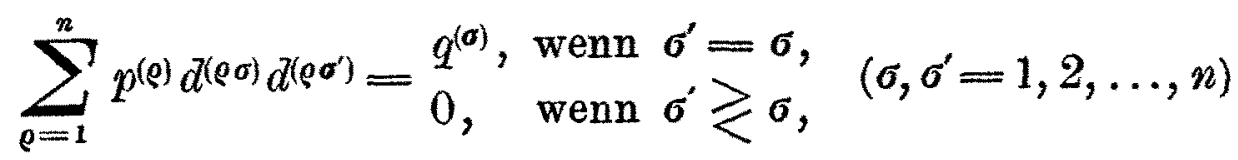

genügen, die zuerst von Herrn $\mathrm{Kraus} \mathrm{e}^{*}$ ) mitgetheilte, und von ihm "Additionstheorem zwischen Thetafunctionen mit verschiedenen Moduln" genannte Formel:

$$
\begin{gathered}
\nabla^{(n-1) p} \vartheta\left(\left(u^{(1)}\right)\right)_{a^{(1)}} \ldots \vartheta\left(\left(u^{(n)}\right)\right)_{a}(n) \\
=\sum_{[\alpha]}^{0,1, \cdots, \nabla^{-1}} \vartheta\left[\begin{array}{c}
\frac{\overline{\bar{\alpha}}^{(1)}}{\Delta} \\
0
\end{array}\right]\left(\left(v^{(1)}\right)\right)_{b}(1) \ldots \vartheta\left[\begin{array}{c}
\frac{\overline{\bar{\alpha}}^{(n)}}{\Delta} \\
0
\end{array}\right]\left(\left(v^{(n)}\right)\right)_{b^{(n)}}
\end{gathered}
$$

dabei ist:

es ist ferner:

$$
a_{\mu \mu^{\prime}}^{(\varrho)}=p^{(\varrho)} a_{\mu \mu^{\prime}}, \quad b_{\mu \mu^{\prime}}^{(\sigma)}=q^{(\sigma)} a_{\mu \mu^{\prime}}, \quad\left(\begin{array}{l}
\varrho, \sigma=1,2, \ldots, n \\
\mu, \mu^{\prime}=1,2, \ldots, p
\end{array}\right)
$$

oder umgekehrt:

$$
v_{\mu}^{(\sigma)}=\sum_{\rho=1}^{n} d^{(\rho \sigma)} u_{\mu}^{(\rho)}, \quad\left(\begin{array}{l}
\sigma=1,2, \ldots, n \\
\mu=1,2, \ldots, p
\end{array}\right)
$$

$$
u_{\mu}^{(\rho)}=\frac{1}{\Delta} \sum_{\sigma=1}^{n} \delta^{(\rho \sigma)} v_{\mu}^{(\sigma)} ; \quad\left(\begin{array}{l}
\varrho=1,2, \ldots, n \\
\mu=1,2, \ldots, p
\end{array}\right)
$$

es ist weiter zur Abkürzung gesetzt:

$$
\overline{\bar{\alpha}}_{\mu}^{(\sigma)}=\sum_{\varrho=1}^{n} \delta^{(\rho \sigma)} \alpha_{\mu}^{(\rho)} ; \quad\left(\begin{array}{l}
\sigma=1,2, \ldots, n \\
\mu=1,2, \ldots, p
\end{array}\right)
$$

es bezeichnet $\Delta$ den Werth, $\nabla$ den absoluten Werth der Determinante $\Sigma \pm d^{(11)} d^{(22)} \ldots d^{(n n)}$ und $\delta^{\left(e^{\sigma}\right)}$ die Adjuncte von $d^{(\rho \sigma)}$ in dieser Determinante; es ist endlich die auf der rechten Seite von (16) angedeutete Summation in der Weise auszuführen, dass für $\begin{aligned} & \varrho=1,2, \ldots, n \\ & \mu=1,2, \ldots, p\end{aligned}$ nach

*) Krause, Ueber Fourier'sche Entwicklangen im Gebiete der Thetafunctionen zweier Veränderlichen. Math. Ann. Bd. 27 (1886), pag. 424, Formel (11). Etwas früher wurde die Formel (16) für den Fall einfach unendlicher Thetareihen und unter der speciellen Annahme $p^{(1)}=p^{(2)}=\cdots=p^{(n)}=1$ von Herrn Krause in seiner Abhandlung: Zur Transformation der elliptischen Functionen. Leipz. Ber. 1886, pag. 39 mitgetheilt. 
jedem $\alpha_{\mu}^{(\rho)}$ ron 0 bis $\nabla-1$ zu summiren ist. Die dabei als Summanden auftretenden $\nabla^{n p}$ Thetaproducte können nach dem in Art. 10 des zweiten Abschnitts Bewiesenen in $\frac{\nabla^{n p}}{\nabla^{(n-1) p}}=\nabla^{p}$ Gruppen geordnet werden, indem man zu einer Gruppe jedesmal diejenigen Thetaproducte, immer $\nabla^{(n-1) p}$ an der Zahl, zusammenfasst, für welche sich die Werthe der $n p$ Grössen $\overline{\bar{\alpha}}_{\mu}^{(\sigma)}\left(\begin{array}{l}\sigma=1,2, \ldots, n \\ \mu=1,2, \ldots, p\end{array}\right)$ nur um ganze Vielfache von $\Delta$ ändern, wenn man ron einem dieser $\nabla^{(n-1) p}$ Thetaproducte zu einem andern derselben übergeht. Die $\nabla^{(n-1) p}$ in einer Gruppe vorkommenden Thetaproducte besitzen dann denselben Werth, und man kann daher in obiger Summe jede solche Gruppe von Summanden durch das $\nabla^{(n-1)} p$-fache eines beliebigen unter ihnen ersetzen. Führt man diese Vereinigung für jede der $\nabla^{p}$ Gruppen aus, so geht die rechte Seite der Gleichung $(16)$ in das $\nabla^{(n-1)} p_{\text {-fache einer Summe }}$ von $\nabla^{p}$ wesentlich verschiedenen Thetaproducten über. Man wird endlich noch bemerken, dass in Folge der aus den Gleichungen sich ergebenden Beziehungen:

$$
p^{(\varrho)} d^{(\varrho \sigma)}=\frac{1}{\Delta} q^{(\sigma)} \delta^{(\varrho \sigma)} \quad(\varrho, \sigma=1,2, \ldots, n)
$$

die Grössen $u$ auch in der Gestalt:

$$
u_{\mu}^{(\rho)}=p^{(\rho)} \sum_{o=1}^{n} \frac{a^{(\rho o)}}{q^{(\alpha)}} v_{\mu}^{(o)} \quad\left(\begin{array}{l}
\varrho=1,2, \ldots, n \\
\mu=1,2, \ldots, p
\end{array}\right)
$$

und die Grössen $\overline{\bar{\alpha}}$ auch in der Gestalt:

$$
\overline{\bar{\alpha}}_{\mu}^{(\sigma)}=\frac{\Delta}{q^{(\sigma)}} \sum_{\varrho=1}^{n} p^{(\varrho)} d^{(\varrho \sigma)} \alpha_{\mu}^{(\rho)} \quad\left(\begin{array}{l}
\sigma=1,2, \ldots, n \\
\mu=1,2, \ldots, p
\end{array}\right)
$$

dargestellt werden können, und endlich sich für das Quadrat der Determinante $\Delta$ der Werth:

$$
\Delta^{2}=\frac{q^{(1)} q^{(2)} \ldots q^{(n)}}{p^{(1)} p^{(2)} \ldots p^{(n)}}
$$

ergiebt.

Lässt man in dex Formel (16) für $\begin{aligned} & \sigma=1,2, \ldots, n \\ & \mu=1,2, \ldots, p\end{aligned} v_{\mu}^{(\sigma)}$ in $v_{\mu}^{(\sigma)}+x_{\mu}^{(\sigma)} \pi i$ übergehen, indem man unter den $x$ ganze Zahlen versteht, und bezeichnet mit $\hat{\hat{x}}_{\mu}^{(\rho)}$ den Ausdruck:

$$
\hat{\hat{x}}_{\mu}^{(\rho)}=\sum_{\sigma=1}^{n} \delta^{(\rho \sigma)} x_{\mu}^{(\sigma)}=\Delta p^{(\rho)} \sum_{\sigma=1}^{n} \frac{a^{(\rho \sigma)}}{q^{(\sigma)} x_{\mu}^{(o)},} \quad\left(\begin{array}{l}
\rho=1,2, \ldots, n \\
\mu=1,2, \ldots, p
\end{array}\right)
$$


so erhält man unter Anwendung der bekannten Hülfsformeln die allgemeinere Formel:

$$
\begin{aligned}
& \vartheta\left[\begin{array}{c}
0 \\
\hat{\boldsymbol{x}}^{(1)} \\
\hline \Delta
\end{array}\right]\left(\left(u^{(1)}\right)\right)_{\alpha}(1) \cdots \vartheta\left[\begin{array}{c}
0 \\
\hat{\boldsymbol{t}}_{(n)} \\
\frac{x^{(n)}}{\Delta}
\end{array}\right]\left(\left(u^{(n)}\right)\right)_{\alpha}(n) \\
& =\sum_{[\alpha]}^{0,1, \cdots, \nabla^{-1}} \vartheta\left[\begin{array}{c}
\frac{\overline{\bar{\alpha}}^{(\mathfrak{1})}}{\Delta} \\
0
\end{array}\right]\left(\left(v^{(1)}\right)\right)_{b}(1) \cdots \vartheta\left[\begin{array}{c}
\frac{\overline{\bar{\alpha}}^{(n)}}{\Delta} \\
0
\end{array}\right]\left(\left(v^{(n)}\right)_{b}(n) e^{\frac{2 \pi i}{\Delta} \sum_{\sigma=1}^{n} \sum_{\mu=1}^{p} \overline{\bar{\alpha}}_{\mu}^{(\sigma)} x_{\mu}^{(\alpha)}}\right.
\end{aligned}
$$

und aus dieser, indem man über jedes $x$ von 0 bis $\nabla-1$ summirt, die Formel:

$$
\begin{aligned}
& \nabla^{p} \vartheta\left(\left(v^{(1)}\right)\right)_{b}(1) \cdots \vartheta \vartheta\left(\left(v^{(n)}\right)\right)_{b}{ }^{(n)}
\end{aligned}
$$

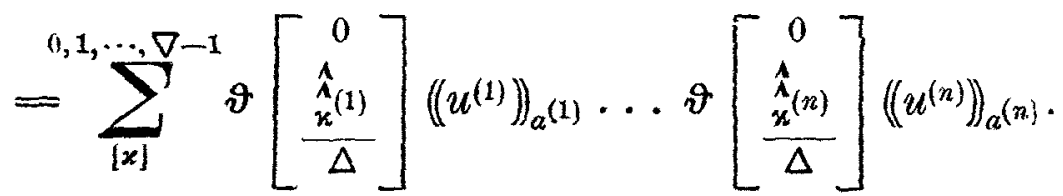

Diese Formel, von der man auch umgekehrt wieder zu der Formel (16) zurückkehren kann, und die daher als von der Formel (16) nicht wesentlich verschieden anzusehen ist*), ist zuerst und viel früher als die Formel (16) von Herrn Gordan ${ }^{* *}$ ) mitgetheilt worden.

Für $r=2$ geht die Formel (II) in das "zweite Additionstheorem" des Herrn Krause $e^{*}$ über.

Was die Bedeutung der Formel (II) angeht, so liefert dieselbe durch Specialisirung der ihr zu Grunde liegenden linearen Substitution und der Argumente der in ihr auftretenden Thetafunctionen eine Fülle von Relationen, welche im Falle der Transformation höheren Grades zwischen den ursprünglichen und den transformirten Thetafunctionen bestehen $\dagger$ ). Die Formel (II) geht aber ferner, wenn man die Modulen

*) Die von Herrn Krause am Ende seiner oben citirten Abhandlung in den Leipz. Ber. 1886 pag. 43 gemachte Bemerkung ist demgemäss za berichtigen.

**) Gordan, Beziehungen zwischen Thetaproducten. J. für Math. Bd. 66 (1866) pag. 189, Formel (VIII). Später als die Herren Krause und Gordan hat die Formeln (16) und (27) Herr Mertens, Deber eine Verallgemeinerung der Schröter'schen Multiplicationsformeln für Thetareihen (Progr. Köln 1889), angegeben.

***) Krause, Ueber Fourier'sche Entwicklungen im Gebiete der Thetafunctionen zweier Veränderlichen. Math. Ann. Bd. 27 (1886), pag. 425, Formel (4).

i) Vergl. hiezu ausser den schon citirten Abhandlungen noch:

Göring, Untersuchungen über die Theilwerthe der Jacobi'schen Thetafunctionen und die im Gauss'schen Nachlasse mitgetheilten Beziehnngen derselben. Math. Ann. Bd. 7 (1874) pag. 311.

Kranse, Die Transformation der hyperelliptischen Functionen erster Ordnung. Leipzig 1886.

Möller, Zur Transformation der Thetafunctionen. Inang.-Diss. Rostock 1887. 
A. Krazer. Ueber allgemeine Thetaformeln.

aller in ihr vorkommenden Thetafunctionen einander gleich, $d . b$. $p^{(1)}=\cdots=p^{(n)}=q^{(1)}=\cdots=q^{(n)}=1$ setzt, in die von Herrn Prym*) mitgetheilte ,allgemeine Thetaformel" über, die nun ihrerseits alle zum Kreise der Additionstheoreme gehörigen Formeln, insbesondere die Riemann'sche Thetaformel und deren Verallgemeinerungen ${ }^{* *}$ als specielle Fälle enthält.

Strassburg i. E., im Januar 1899.

Krause, Zur Transformation der Thetafunctionen. Leipz. Ber. 1893, pag. 99, 349,523 and 805 ; 1896, pag. 291.

Krause, Ueber die Transformationstheorie der elliptischen Functionen. Jahresb. der d. Math. Ver. Bd.4 (1894-1895), pag. 121.

Krause, Theorie der doppeltperiodischen Functionen einer veränderlichen Grôsse. Leipzig. Bd. 1 (1895), Bd. 2 (1897).

*) Prym, Ableitung einer allgemeinen Thetaformel. Acta math. Bd. 3 (1883), pag. 216.

**) Krazer und Prym, Neue Grundlagen etc. pag. 33 a.f. and pag. 47 u.f. 